\title{
Images of the arXiv: Reconfiguring large scientific image datasets
}

Kynan Tan, Anna Munster, and Adrian Mackenzie

Kynan Tan. University of New South Wales. kynan.tan@unsw.edu.au.

Anna Munster. University of New South Wales. a.munster@unsw.edu.au.

Adrian Mackenzie. Australian National University. adrian.mackenzie@anu.edu.au.

Peer-Reviewer: Richard Rogers, Greg Mclnerny

Dataverse DOI: 10.7910/DVN/EAAG94

ABS TRACT

In an ongoing research project on the ascendancy of statistical visual forms, we have been concerned with the transformations wrought by such images and their organisation as datasets in 're-drawing' knowledge about empirical phenomena. Historians and science studies researchers have long established the generative rather than simply illustrative role of images and figures within scientific practice. More recently, the deployment and generation of images by scientific research and its communication via publication has been impacted by the tools, techniques, and practices of working with large (image) datasets. Against this background, we built a dataset of 10 million-plus images drawn from all preprint articles deposited in the open access repository arXiv from 1991 (its inception) until the end of 2018. In this article, we suggest ways - including algorithms drawn from machine learning that facilitate visually 'slicing' through the image data and metadata - for exploring large datasets of statistical scientific images. By treating all forms of visual material found in scientific publications - whether diagrams, photographs, or instrument data - as bare images, we developed methods for tracking their movements across a range of scientific research. We suggest that such methods allow us different entry points into large scientific image datasets and that they initiate a new set of questions about how scientific representation might be operating at more-than-human scale.

\section{Introduction}

In an ongoing research project ${ }^{1}$ on imaging and machine learning, we have been concerned with how the ascendancy of statistical visual forms - particularly from the 1990s on ${ }^{2}$ - has come to transform and re-organise images so as to 'redraw' knowledge about empirical phenomena. Historians and science studies researchers have long established the generative rather than simply illustrative role of images and figures within scientific practice. ${ }^{3}$ More recently, the use of images within computer science specifically has garnered attention: "[they] often contain a crucial part of the information scholarly documents convey. Authors frequently use figures to compare their work to previous work, to convey the quantitative results of their experiments, or to provide visual aids to help 
readers understand their methods." ${ }^{4}$ One distinction that has run through this debate is the difference between scientific images used to authorise and consolidate the truth claims and/or authority of experiments or data, which tend to be diagrammatic, and images that are reproductions of scientific evidence. ${ }^{5} \mathrm{~A}$ division is thus made between images that perform a transformation upon data and ones that are merely representations of that data.

While this debate about the status of images in science has occurred over the last 50 years, the deployment and generation of images by scientific research and the communication of research via publication has been more recently impacted by the tools, techniques and practices of working with large (image) datasets. The standout example here would be the release in 2009 of the ImageNet dataset along with the spawning of a culture of specific computer vision research in the form of object recognition challenges, which, up until 2017 when it ended, had students and staff, industry R\&D teams as well as lone 'self-employed' challengers participating. ${ }^{6}$ This shift, exemplified by the production of a large scale image dataset that quickly becomes the object of and for research in a field, is concomitantly accompanied by the techniques, technologies, and practices that are required to turn the data into knowledge about the empirical world. Advancing in tandem with such image datasets are machine learning (ML) algorithms, processes of optimisation of the data that utilise ML, and indeed entire models that amass different statistical processes interfacing with ML for analysing and sometimes predicting from the dataset inputs. In everything from face recognition in crowds, SARS-CoV-2 detection in chest X-rays, or in weapons targeting platforms, images themselves become the means through which ML models the statistical arm of artificial intelligence - input 'information' about the world, vertiginously contouring and hence 're-drawing', in the sense of reconfiguring, the landscape of 'knowing.' ML models have come to use large datasets such as ImageNet as training material, learning weights and biases that enable the model to then classify previously unseen images. At the same time, ML practice relies on and is communicated through figures, especially since many of its statistical processes are 'black-boxed.'7 
We set out using an empirical and exploratory approach by suspending the separation of the performative from the reproductive scientific image. We track, instead, observable differences in how images circulate through scientific research. By treating all forms of visual material found in scientific publications - whether diagrams, photographs, or instrument data - as bare images, we developed methods for tracking their movements across a range of scientific research. In many ways this is made possible by the labelling of images within scientific publications as 'figures.' Accordingly we treated all material appearing under the 'figure' caption as such 'bare' images. As we tracked these movements, we were better able to ask: what specific forms do the 'output' images, produced via a range of techniques, including statistical ones, assume? In what ways is the input (image) data transformed so as to become specific kinds of visual figures, newly configuring and indexing empirical phenomena? Moreover, we also sought to ask: how might we come to see the circulation of such visual figures across the vast corpus of scientific work? In this article, we detail the dataset and statistical exploratory methods deployed to trace this circulation of visual forms. We suggest that such methods allow us different entry points into large scientific image datasets and that they initiate a new set of questions about how scientific representation might be operating at more-than-human scale.

Before we outline and discuss these methods, a couple of notes concerning the conceptual and theoretical background against which we have developed these methods. First, we think about scientific images in the context of the welldeveloped discussion of 'inscriptions' in science studies. ${ }^{8}$ Here images that are generated (and other techniques for inscribing scientific information including publishing), and that appear and circulate throughout all modes of scientific practice, do not simply represent data or 'facts.' Their visual nature or indexical status is not what is primarily of importance. Instead inscriptions primarily perform two functions: the mobilisation of scientific objects among scientific communities of practice; and the consolidation and authorisation of the scientificity of such objects. This gives inscriptions a paradoxical status of being both mover-shakers and stabilisers of scientific research and practice. Much 
work has been done in visual science studies to look at how specific kinds of imaging techniques and technologies work to authorise claims about scientific data but, at the same time, destabilise and challenge knowledge formation. ${ }^{9}$ In our work, we similarly focus on what distributed circulation across platforms and networks, as a peculiarly contemporary mode of mobilisation, might tell us about the current status and recent history of a heterogeneous array of statistical visual forms. We are less concerned, then, with what these forms empirically represent than in what operations they perform in bringing empirical phenomena into the dispersed infrastructures of scientific knowing - repositories, preprints, formatting of image files, to name only a few.

However, we are not primarily concerned with how images circulate within a specified community of scientific practice nor in how such images/inscriptions help to sustain such communities. In part, this is because in tracing the circulation of images across the sciences we enter an arena in which the vast quantities of such images moving online and across platforms do so at a scale before and beyond specific institutional and lab-based communities of scientific practice. ${ }^{10}$ This migratory massification of images had already been heralded by the production of large commercially available GIS datasets from the 1980s onward. But the scale and availability of public image datasets and their use in ML practices such as image recognition challenges really took off in the 2000s. Importantly, then, we are no longer talking about how inscriptions are mobilised but rather how an entire assemblage - the dataset - becomes an operative apparatus. In this contemporary situation, we look to a critical, even post-, digital humanities ${ }^{11}$ for experimental approaches to large scientific datasets. We deploy computational methods that draw on ML itself but that also allow us to develop a situated and interrogative perspective on the circulating, exchanging, and associative tendencies of large aggregations and flows of statistical image forms. 


\section{(The making of a large scientific image dataset}

A crucial prerequisite of this work has been to generate a dataset of images drawn from scientific research that manifests the knowledge claims about empirical phenomena. Such knowledge claims are now entangled with the authority that statistical approaches have found in sciences, which also deploy data methods. ${ }^{12}$ How do these statistical approaches also make their way into images? We built a dataset of images drawn from all preprint articles deposited in the open access repository arXiv from 1991 (its inception) until the end of $2018 .^{13}$ ArXiv (pronounced "archive") maintains over 1.5 million e-print articles across a diverse range of scientific fields. Created in 1991 by physicist Paul Ginsparg, arXiv provides a platform for authors to share articles prior to or during the process of peer-review. ${ }^{14}$ Researchers in many fields, such as high energy physics, rely on arXiv as their primary source for accessing current scholarship. ${ }^{15}$ The number of submissions to arXiv has been growing in recent years, with 140,242 new articles added to the repository in $2018 .{ }^{16}$

ArXiv stores papers across a range of knowledge domains from the sciences including but not limited to: physics, mathematics, statistics, computer science and subfields of biology and economics. ${ }^{17}$ Its development as a repository also maps many key developments for images and image-related tasks that use statistical approaches, in particular ML. To name only a few: the shift within astrophysics to data management and the classification, segmentation and recognition of objects across a new scale of image datasets enabled by the launch of the Hubble Telescope in 1990; the release of the MNIST handwriting dataset in 1998; the creation of ImageNet in 2009; Google Brain's 2012 deep neural network architecture for recognising cat images from unlabeled images taken from frames of YouTube videos; and the 2016 AlphaGo model, trained on millions of 19x19 pixel images of Go board states. The images of arXiv - as elements within the research papers of these and many more ML projects and developments - document a growing statistical visuality, as a technical practice which is concerned with the statistical observation of something in data. 
ArXiv provides a relatively accessible bulk download procedure for its articles. ${ }^{18}$ From this bulk download, we were able to access all papers (and associated images) uploaded to arXiv from its inception to the end of 2018. Articles uploaded to arXiv consist of either: solely a PDF file (7.69\%); TeX/LaTeX source code only (21.95\%); or TeX/LaTeX source code with figures as separate image files $(70.32 \%) .{ }^{19}$ ArXiv provides multiple modes of accessing the repository: to the articles themselves via a web interface with download links; to their metadata via an OAI-2 interface; and to bulk source or PDF downloads via Amazon Web Services. We used the OAI-2 metadata and the bulk source data together to construct our dataset with metadata indexing. As an open access repository status within scientific publishing and research distribution - particularly within fields that might have interest in source material for large datasets such as computer science and statistics - arXiv has not unsurprisingly already been used as source data. However, prior dataset engagement has almost exclusively used its text data, deploying this for citation tracking, natural language processing, or topic tracking and prediction. ${ }^{20}$ Across the arXiv dataset there is a mean average of over seven images per paper. Yet very little research has been conducted into using arXiv as a source for querying or analysing its image data. $^{21}$

Completing the download in January 2019 provided us with over 10 million images for our arXiv image dataset. What could an image collection structured as a dataset comprising diagrams, photographs and other graphic elements drawn from the range of scientific fields present in arXiv tell us about how statistical computing practices such as ML reconfigure ways of seeing and knowing real world phenomena? How might we slice through a highly diverse and voluminous image dataset, engaging with its emergent forms of organising and circulating statistical image 'types' without simply classifying or segmenting the images according to an uninterrogated taxonomy of visual forms: photographs, scatterplots, feature maps, bar graphs and so forth? The rationale behind the decision to use arXiv was that it would provide us with a dataset that contained a recent historical record of the ways in which images have figured in scientific 

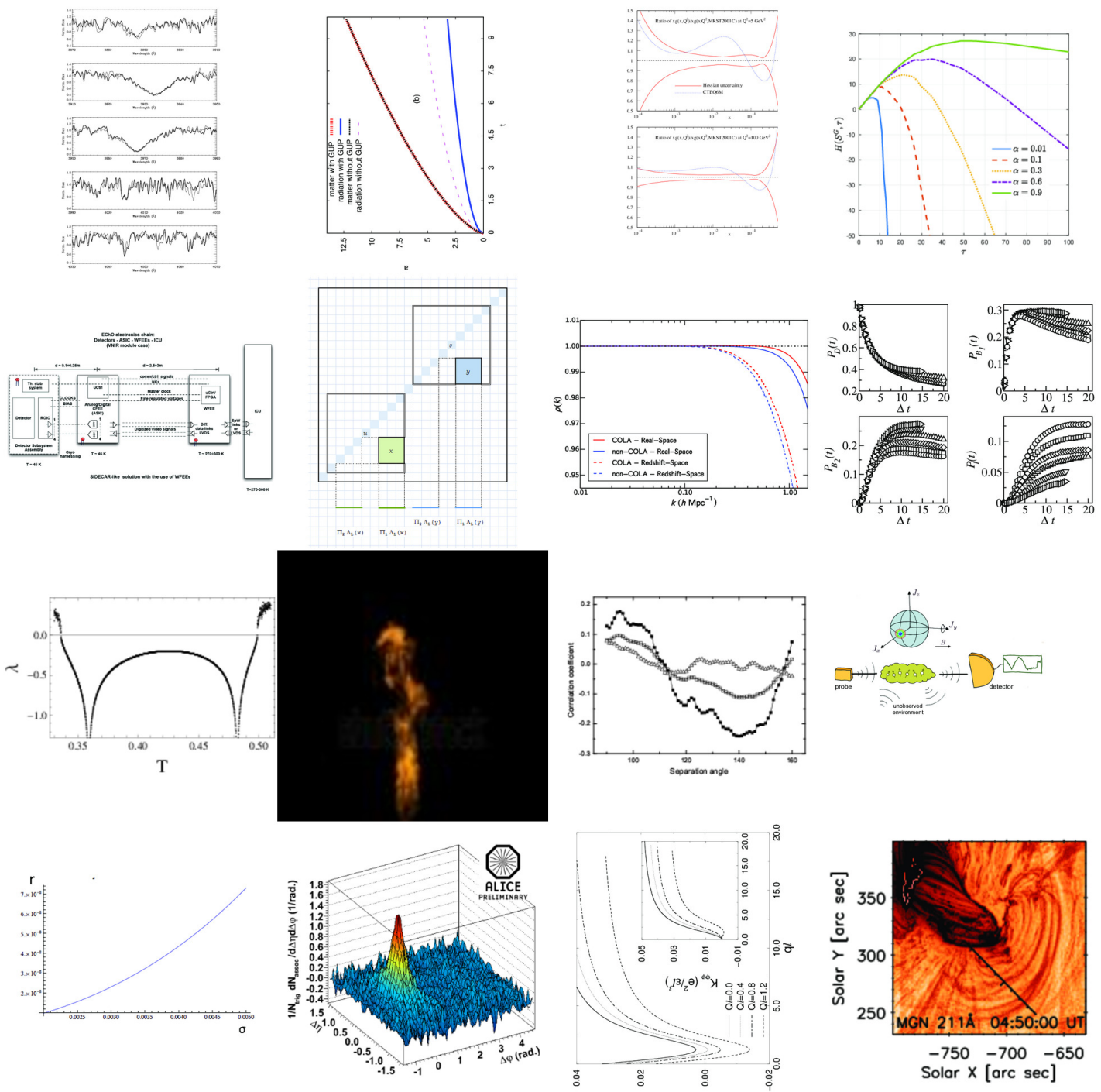

Figure 1: Montage of 16 images sampled randomly from the arXiv image dataset. Images have been resized to fit within a 480x480 pixel square. Seen here are a number of plots, charts and graphs, as well as other data visualisations, processed sensor imagery and computer synthesised images.

* Source materials uploaded to arXiv are either given a specific licence (such as creative commons) or use the standard arXiv distribution licence, which grants arXiv permission to distribute the article/images but the creator retains copyright. This licence remains with the images when downloaded and used by other parties. This is particularly important as the authors of a paper may not have the rights to distribute the images, such as in the case of photographs that are being processed by computer vision algorithms. In the interest of properly accrediting the creators of these images, we have created a website which provides accreditation for each image at https://github.com/re-imaging/re-imaging/blob/master/methods/credits.org. 
scholarship broadly, and ML research specifically. We posited that ML techniques, used in an exploratory and interrogative mode, might provide a means for experimenting with the tendencies and relations of scientific images during a recent historical period in which ML has itself widely affected the production of scientific knowledge in many fields. Additionally, we were interested in the availability of the metadata uploaded through the arXiv submission process. This included standard information about author, article abstract and so forth but also detailed information about the software used to create images. We posited that examining this metadata through dataset querying could unfold information about the situated knowledge contexts out of which these images arise.

We queried the arXiv image dataset according to some of the categories under which preprints were submitted - for example, 'cs.CV,' arXiv's abbreviation for computer vision. The purpose of this was to explore how over the 28 years of arXiv's growth as a repository the images being used within an area of scientific research communication might reveal changes and continuities in the visual cultures of these scientific endeavours. As we have already noted, scientific imaging has undergone many changes over three decades. However these changes are not simply the result of new technological developments within a particular field of research. Rather, we have witnessed an entirely new approach to the organisation, deployment and operations of images in the sciences. While there has been a huge uptake of images as data within varied areas of scientific research, there has been little investigation by either STS or visual studies as to what sociotechnical relations or implications this might have. ${ }^{22}$ In our exploration of the arXiv dataset, we were especially drawn right from the start to the category of computer vision as an area of increased publication submission by arXiv authors, which historically tallies with both the rise of this as a research field; and as one concerned with and deploying statistical imaging processes and methods.

It is clear that within articles communicating scientific research such as those found within arXiv, images cannot simply be understood instrumentally or illustratively. ${ }^{23}$ Specific images within our dataset circulate and are re-used across 
the period of arXiv's development, perhaps suggesting that only partial 'views' of the world may circulate throughout specific communities of practice. Looking at the images within categories of publication such as computer vision within arXiv gives some indications of imaging practices within the empirical sciences and mathematics that have steadily adopted statistical approaches over the last 28 years. It certainly does not provide a definitive contouring of a singular visual culture or mode of working with images as a generalised tendency within research that utilises statistical imaging. But it can highlight some of the tendencies, frictions, and commonalities across seemingly diverse research areas.

The images within the bulk download of arXiv preprint manuscripts lack metadata annotating their scientific function within the preprint. Although the preprint publications have been structured and organised in particular ways - according to the authors' uploads, arXiv guides and requirements, subject categories, and discipline-specific customs, which we detail later in this article - the images within them yield a heterogeneous aggregate that renders their (scientific) function opaque. They contain artifacts of experiments or observations, images captured by sensors, images found in ML datasets and images that are the outputs of ML processing, diagrams and drawings conveying the methods and experimental results undertaken, and diagrams/images that are transformations of the data that convey the 'results' of research.

We hope here to convey some sense of how a 'workable' image dataset can be generated from its source data. We want to give an account, too, of the problems and frictions that present themselves when trying to access, organise and query this data. We also discuss our experiments with various ML techniques, repurposed here to map relations of proximity (similarity) and distance (difference) in the arXiv image dataset. We are interested in whether these relations render clusters of images that can be associated with different imaging styles or forms that then map on to specific knowledge domains signalled by arXiv's 'categories.' This can then be used to compare with, for example, increases in quantities of images per article within a particular category of arXiv publication. It should be stressed that this only produces a correlation: increased quantities 
of images published in a particular category such as computer vision with a potential shift in kinds of images in that same category, for example more sensorbased images appearing in that category over time (this is further discussed below). But before getting to this point we paid attention to quantitatively and qualitatively relating the arXiv image dataset to the metadata accompanying the preprint articles. This allowed us to observe the formatting and generative software deployed in the production of images in the (hard) sciences. Further to this, we have been interested in the distribution of types of images across the entire preprints downloaded and the distribution of such types within particular arXiv knowledge/discipline categories. By types, we mean a pragmatic ternary classification we applied to the dataset using human visual analysis and ML processes. This process distinguished between: 'diagrams,' 'sensor-based images,' and 'hybrid diagram-sensor' or 'mixed' images.

We are proposing that this conjunction of data points and techniques produces potential amplifications, resonances, and disjunctions across a large scientific image dataset, suggesting tendencies in their distribution and circulation across domains of scientific knowledge and practice. In downloading, organising, converting, querying, and sampling from this dataset we also highlight the particular challenges and difficulties of working with any large dataset. By outlining the steps taken to work with and gain some understanding of the data, we hope to give an account of some dimensions of data research that may often be rendered invisible, opaque or even unworthy of attention in many domains producing knowledge through their use of 'big data.' We consider all aspects of dataset methods important contributors to data work, and are interested in elucidating the various exploratory data practices and processes that emerge when working with data from the bottom up.

Without denying the significant communicative functions of scientific images, we also consider how images in aggregate, as a large collection, might do something more. The dataset presents a number of characteristics that we believe inhibit widespread use or distribution as a dataset. First, the dataset is large (2.1 terabytes across 1.5 million article folders) and requires a number of steps to 
acquire and organise, while also being difficult to sample data subsets. Second, the formats of its images are inconsistent. There are a wide variety of image formats ranging from standard photographic (TIFF, JPEG) and web formats (PNG, GIF) to vector graphics (SVG), postscript (EPS, PS). Third, images in the dataset have varying dimensions, ratios, and image quality. Lastly, not all images that are used in arXiv preprints are available in source form and cannot be consistently retrieved from PDF articles. We outline some steps we have taken to mitigate and/or work within these constraints.

\section{Structuring arXiv images as a dataset}

The resources, time, and effort required to generate and structure new large datasets from bottom up are frequently a barrier to the diversity of datasets in, especially, ML research. Hence certain standardised image datasets tend to become benchmarks by default. ${ }^{24}$ Instead of using a standard dataset, we embarked on creating this dataset with the idea that by engaging with actual data practices and ML techniques for images, we would, step by step, learn more of the computational operations with which data science engages images and vision. ${ }^{25}$

Table 1: Primary image file extensions, converted to lowercase, with similar extensions combined (e.g. .jpeg and .jpg).

\begin{tabular}{rrr}
\hline extension & total & $\%$ \\
\hline eps & 4223083 & 42.00 \\
pdf & 3299043 & 32.81 \\
png & 1076731 & 10.71 \\
ps & 909314 & 9.04 \\
jpg & 485452 & 4.82 \\
pstex & 23922 & 0.23 \\
gif & 19054 & 0.18 \\
svg & 12400 & 0.12 \\
epsf & 4060 & 0.04 \\
\hline total & 10053059 & 100 \\
\hline
\end{tabular}


We downloaded the full allotment of source files as of December 31, 2018 a total download of approximately 1 terabyte in the form of 2150 compressed tar archive files. ${ }^{26}$ Using an exploratory approach, our data practices shifted in response to the particular structures and challenges of the dataset. This required a recursive approach to the dataset as something whose structuring required updating as we explored and analysed the image, text, and metadata. This required us to do some initial steps of downloading, organising, and exploring the data in order to ascertain the file formats that appear commonly across the bulk dataset before finalising how to organise and extract the images. For example, we observed that the ways that image data has been submitted and stored in arXiv diverges historically and in terms of topics of research within categories. A shift in image formats occurs from almost entirely PostScript vector graphics formats (.ps and .eps) until around 2006, to PDFs and web image formats (.jpg, .png) assuming the larger proportion of formatting by the mid 2010s and then dominating in the late 2010s (see figure 2). This tracks a change from formats utilised primarily for printing to those captured by digital cameras and/or distributed via the internet. Although this shift in image formats may appear to be a minor issue for building a dataset - commonly understood as an aspect of data cleaning within data science research - for us it also converges with broader events in the surge in and spread of ML and its increasing dependence on image data to build algorithms and indeed entire models.

The use of web image formats (.jpg and .png) for images embedded in preprints is particularly interesting since it might suggest that such images are already in online circulation prior to becoming figures in a scientific paper. This may simply be because such images have already appeared in research blogs or online conference proceedings. But it also suggests that images that are on the web exist as scrape-ready for scientific research; that is, able to be downloaded en masse. The potential for web formatted images to become data resources is also aided by the fact that many social media platforms that use web formatting for their images also provide APIs that allow for external image collection matched to labels. The well known case of IBM's 99.2 million webscrape of Flickr to 


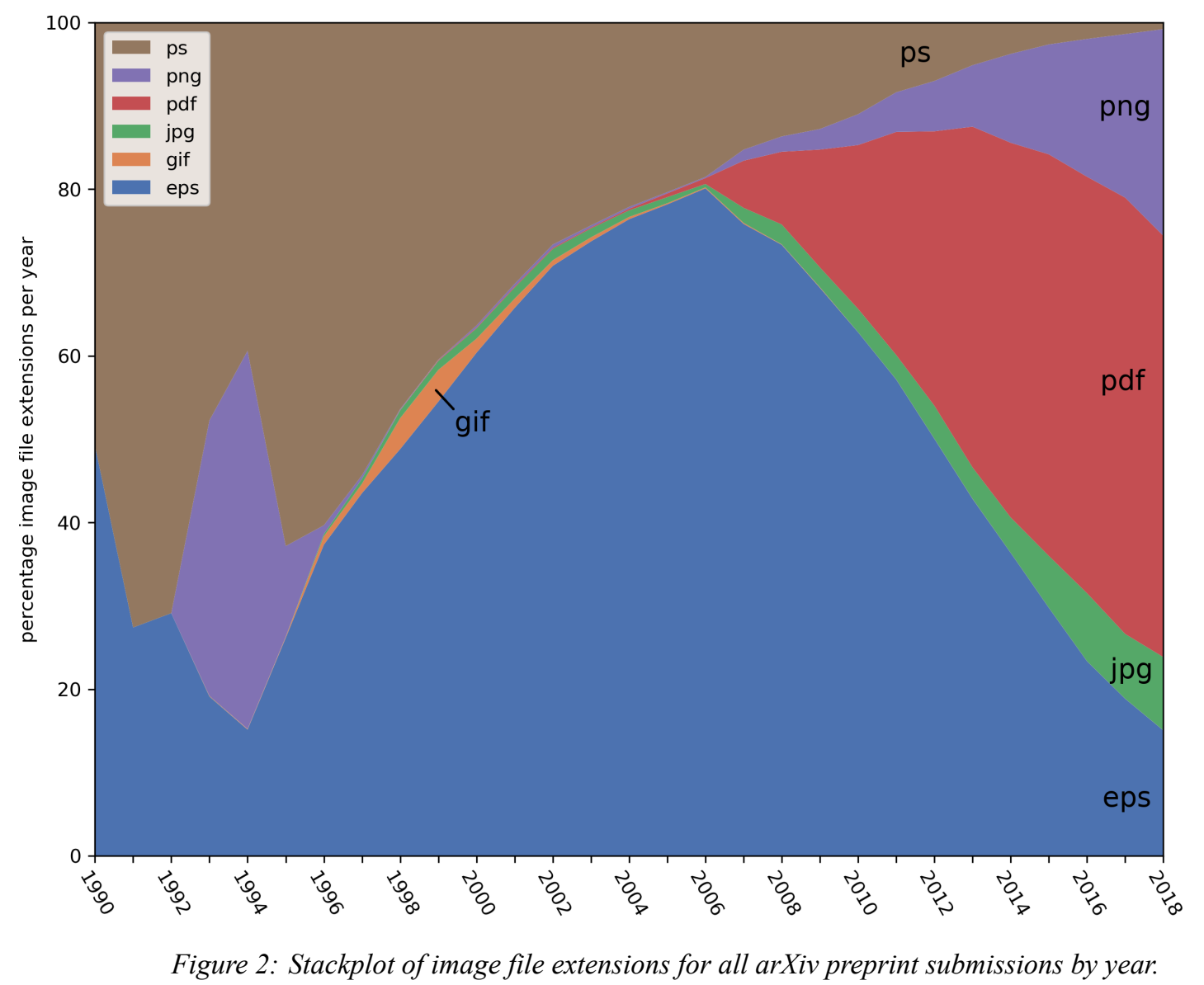

produce its facial image dataset 'Diversity in Faces' is a case in hand. ${ }^{27}$

The PDF format also tells a story about the circulation and distribution of image data (or for that matter, text, fillable forms, pop-up comments, video and audio material, semantic tags and so on). The key characteristics of the PDF format in the context of this research is its standardisation, which makes it cross-platform reliable and queryable. ${ }^{28}$ What we would suggest, then, is that the shift in formatting to both web-based and standardised formats for images in arXiv from 2010 onwards indexes and contributes to a broader shift in the status of images as potential data source. That is to say, while image content and its communicative function retains a degree of importance for scientific publication, they by no means exhaust the productive capacity of arXiv's images. Instead, arXiv's image formats attest to images as vectors for mass collection, exchange, distribution, and storage. In another context, Jonathan Sterne has coined the 
term 'perceptual technics' to foreground how media channels and storage can be economised so as to grab corporate share of a perceptual modality such as hearing. ${ }^{29}$ The shift of image formatting to web-based and cross-platform also charts the transformation of seeing images from something that is human-based to a form of seeing that is now organised via operations of data processing. In our approach to structuring arXiv images as a dataset, we have spent time on aspects such as data extraction and sampling in order to see how data points might resonate with sociotechnical events.

ArXiv bulk source data is separate from article metadata, requiring additional steps to link image data to article metadata such as author, category, or publication date. In order to index the images with their associated article metadata, we created a SQLite database with three tables: metadata with rows for each article; images with rows for each image file (see table 2); and captions for text related directly to images. For metadata, we used a primary key of a unique number, and inserted the identifier, date created, categories, authors, title, abstract, and licence.

Table 2: Example of metadata associated with individual image files.

\begin{tabular}{rll}
\hline & example 1 & example 2 \\
\hline id & 4876126 & 2209549 \\
identifier & cs0007002 & 0906.0725 \\
filename & gouala05.eps & belleescan_b.eps \\
filesize & 145239 & 842045 \\
path & $. / 0007 /$ cs0007002 & $. / 0906 / 0906.0725$ \\
$x$ & 663 & 1450 \\
$y$ & 300 & 725 \\
imageformat & PS & PS \\
\hline
\end{tabular}

The vast majority of image files within arXiv contain metadata relating to the software that created or last exported that image. For each image in the dataset, we ran a query using exiftool, which reads the EXIF (Exchangeable Image File Format) metadata. After some initial testing, it was found each image generally had data written in either the "Creator," "Software," "Comment" or "Desc" 
field that related to software or applications used to edit or create the image and so this was also added. Once both tables have been created, it is possible to perform SQL queries that pair the associated metadata with a given image. This allows us to create queries and perform analyses where the image data can be linked to subject categories or date. ${ }^{30}$

\section{Querying and analysing the image dataset}

ArXiv provides some interesting statistics concerning submission to the repository over time and by category. ${ }^{31}$ We supplement these with statistics that are specifically about the images held in the repository (see table 3). We have also used arXiv user submission statistics in the choices we made in the image sampling. For example, noting the peak periods of publication dates across October and November in 2018, we used articles submitted in October 2018 from which to sample a larger range of images. At a later point in this project, it may be possible to correlate or compare data points across years and categories more widely.

Table 3: Metadata statistics for arXiv image dataset. Calculated after filtering data for primary image formats. The given percentages refer to the proportion of this total number of images.

\begin{tabular}{rrr}
\hline Total articles & $1,476,538$ & \\
\hline Articles with at least one image & $1,038,305$ & $(70.32 \%)$ \\
PDF-only articles & 114,132 & $(7.72 \%)$ \\
TEX/LTEX source code only $_{\mathrm{E}}$ (I) & 324,101 & $(21.95 \%)$ \\
\hline Total number images & $10,053,059$ & \\
\hline
\end{tabular}

Across the 1.47 million arXiv papers, $70.32 \%$ contain at least one image. Of these articles with at least one image, the average number of images is 9.68. Table 4 shows the "creator" field in the metadata of image files. The largest single result from this is $8.72 \%$ of images being created by some version of MATLAB, with Mathematica and Matplotlib also frequently used. The majority of the most commonly used software formats generate images programmatically, 
which suggests a direct connection between data processing and figure generation. Of the top results, Adobe Illustrator (2.63\%), GIMP (GNU Image Manipulation Program, 2.06\%), Adobe Photoshop (0.76\%), Inkscape (0.32\%), and perhaps OmniGraffle $(0.31 \%)$ suggest more manual image editing via a graphical user interface. PowerPoint $(0.50 \%)$ and Keynote $(0.38 \%)$ suggest using presentation software to either create or export images. The other results show a diverse mix of software applications, command line tools, and plugins that may be used to generate postscript files. Such metadata suggests software and data practices within specific knowledge communities in the sciences pointing toward increased usage of scripted or automated image generation, particularly through the use of scripting or programming languages. 
Table 4: Top results for image metadata in the "creator" field across all image files in the dataset. Metadata cleaned and collated. Percentage refers to the proportion of total number of images. Only entries with greater than 20k images shown. For full results of this data by year, see full creator table.

\begin{tabular}{|c|c|c|c|}
\hline "creator" & total & $\%$ & application type \\
\hline none & 1997457 & 19.87 & - \\
\hline MATLAB & 876177 & 8.72 & computing system \\
\hline Mathematica & 492318 & 4.90 & computing system \\
\hline matplotlib & 491001 & 4.88 & plotting library \\
\hline IDL & 404852 & 4.03 & programming language \\
\hline gnuplot & 396484 & 3.94 & graphing utility \\
\hline cairo & 388108 & 3.86 & graphics library \\
\hline fig2dev & 349381 & 3.48 & conversion utility \\
\hline SM & 268902 & 2.67 & plotting program \\
\hline ROOT & 265278 & 2.64 & data analysis framework \\
\hline Illustrator & 263934 & 2.63 & vector graphics editor \\
\hline Grace & 237719 & 2.36 & plotting tool \\
\hline dvips & 232165 & 2.31 & conversion utility \\
\hline $\mathrm{T}_{\mathrm{EX}}$ & 209613 & 2.09 & typesetting system \\
\hline GIMP & 207108 & 2.06 & manual image manipulation \\
\hline Ghostscript & 199064 & 1.98 & postscript rendering software \\
\hline OriginLab & 168350 & 1.67 & data analysis software \\
\hline HIGZ & 144720 & 1.44 & graphics interface \\
\hline $\mathrm{R}$ & 143164 & 1.42 & programming language \\
\hline PGPLOT & 128704 & 1.28 & graphics library \\
\hline ImageMagick & 123697 & 1.23 & image software suite \\
\hline CorelDRAW & 91453 & 0.91 & vector graphics editor \\
\hline jpeg2ps & 87546 & 0.87 & image conversion utility \\
\hline PScript5 & 77136 & 0.77 & graphics renderer \\
\hline Photoshop & 76648 & 0.76 & manual image manipulation \\
\hline Acrobat & 72191 & 0.72 & PDF manipulation \\
\hline PowerPoint & 50187 & 0.50 & presentation software \\
\hline XV & 47320 & 0.47 & image manipulation \\
\hline Ipe & 43498 & 0.43 & vector graphics editor \\
\hline Keynote & 37964 & 0.38 & presentation software \\
\hline xmgr & 37831 & 0.38 & plotting tool \\
\hline PSCRIPT & 36755 & 0.37 & postscript printer driver \\
\hline inkscape & 32036 & 0.32 & vector graphics editor \\
\hline OmniGraffle & 30788 & 0.31 & manual diagram editor \\
\hline LATEX & 30473 & 0.30 & typesetting system \\
\hline Preview & 24770 & 0.25 & image viewer and editor \\
\hline GraphicConverter & 24124 & 0.24 & image viewer and editor \\
\hline FreeHEP & 23621 & 0.23 & vector graphics library \\
\hline
\end{tabular}




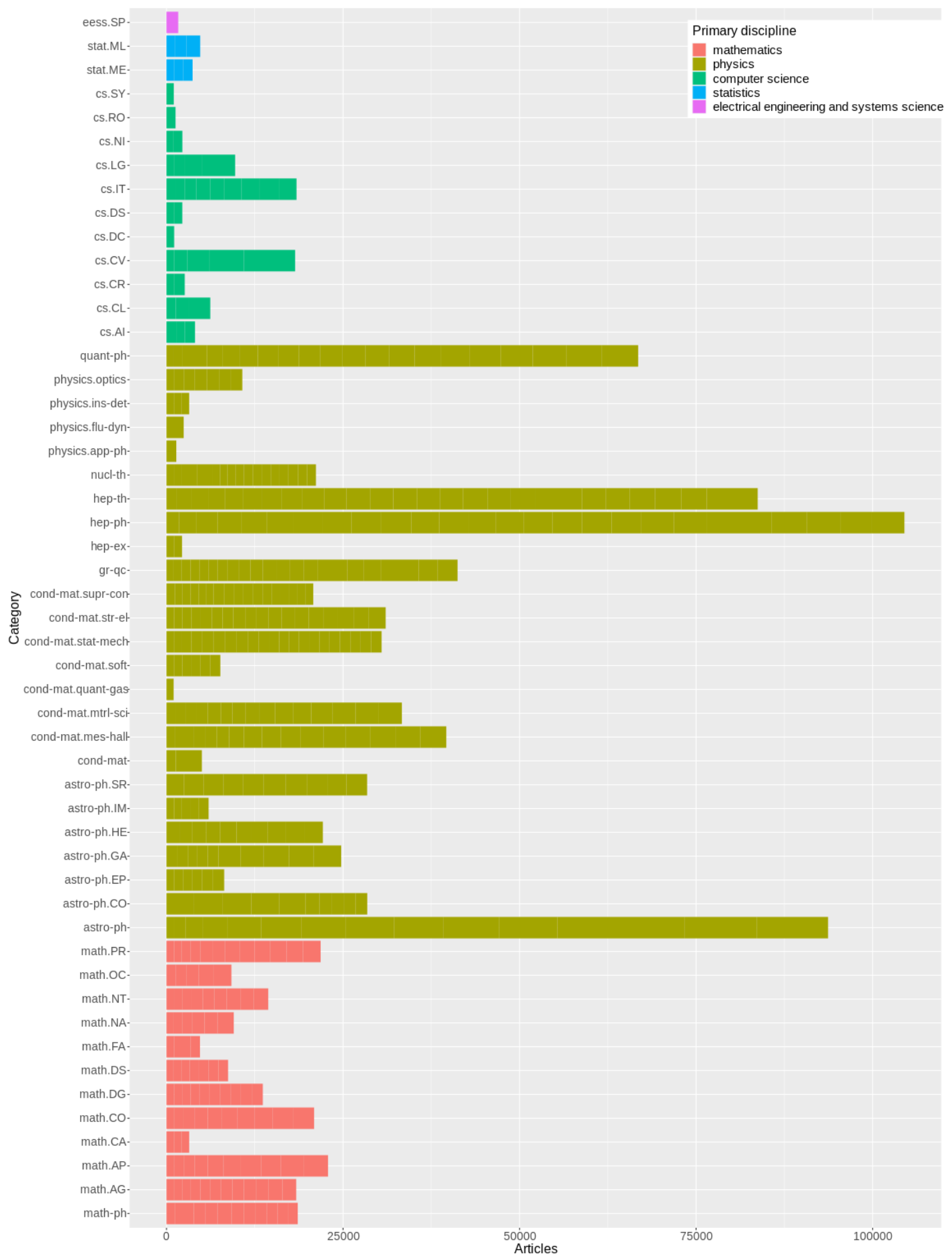

Figure 3: Relative number of articles per arXiv primary category, 1991-2018. Only categories with article counts $>1000$ shown.

Prior to thinking about the distribution of images across categories in arXiv, we 
wanted to get a sense of the overall rates and quantities of preprints being submitted in different disciplines (categories) across the whole of arXiv's life as a repository. Figure 3 shows the number of articles in each primary category, gleaned from querying the arXiv bulk download. There are four categories with significantly higher numbers of articles: hep-ph (Physics: High Energy Physics - Phenomenology), astro-ph (Physics: Astrophysics), hep-th (Physics: High Energy Physics - Theory), and quant-ph (Physics: Quantum Physics). This is unsurprising given the origins of arXiv, which began as a physics preprint repository. ArXiv's own statistics demonstrate the high participation of these disciplines in submission to the platform. Paper submissions are primarily spread between physics, computer science, and mathematics, with the incoming rate for 2019 at $40.9 \%$ physics, $27.8 \%$ cs, and $22.5 \%$ math. $^{32}$

Figure 4 shows the relative percentage of publications submitted in a given category across years from 1991-2018. This figure shows how certain fields have remained relatively consistent, such as many of the math disciplines, whereas other fields spike or grow exponentially in recent years. Computer Science disciplines appear to have the largest growth in the late 2010s, shown by cs.CV (Computer Science: Computer Vision), cs.LG (Computer Science: Machine Learning) or cs.RO (Computer Science: Robotics). Figure 4 shows only relative growth and is of course highly dependent on a number of factors such as the overall use of arXiv and the specific discipline participation habits for sharing preprints via this platform. However, it indicates a trend of increased preprint publishing in several disciplines that deploy or target ML in and for their research.

Figure 5 shows the average number of images per article for the top-16 categories, ordered by largest number of images. There is significant growth in the number of images, particularly in recent years. The most striking subplot is the exponential increase in the number of images per article in preprints submitted to cs.CV (Computer Science: Computer Vision), as well as a significant upwards trend for average number of images in preprints in cs.LG (Computer Science: Machine Learning). We could speculate that this is connected to the 


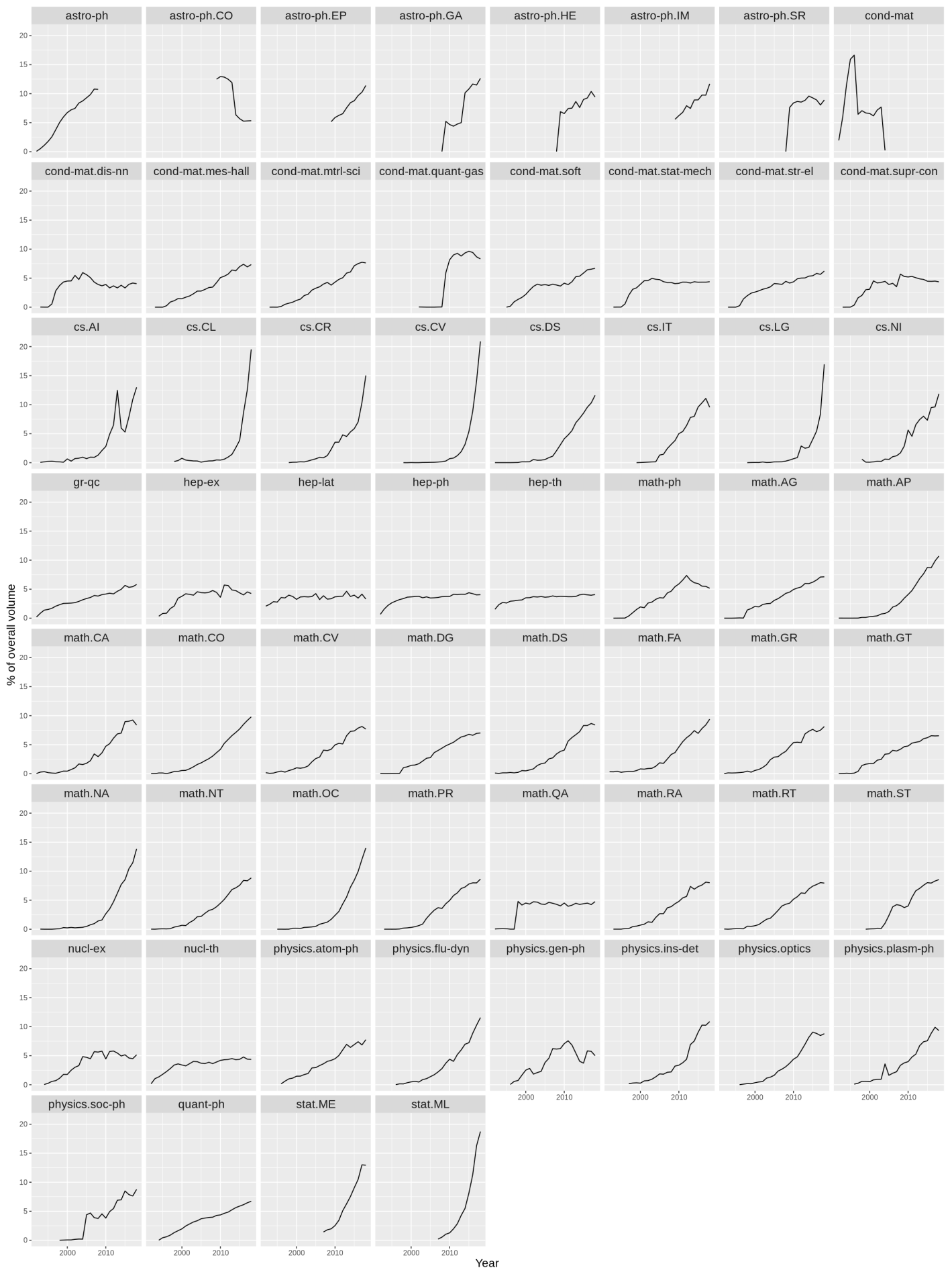

Figure 4: Percentage of articles published by year in a given category, 1991-2018. Top-60 categories by article count shown. For the full list of categories refer to http://arxitics.com/help/categories. 


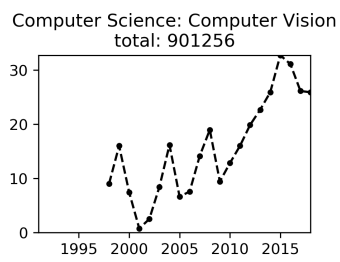

Computer Science: Machine Learning
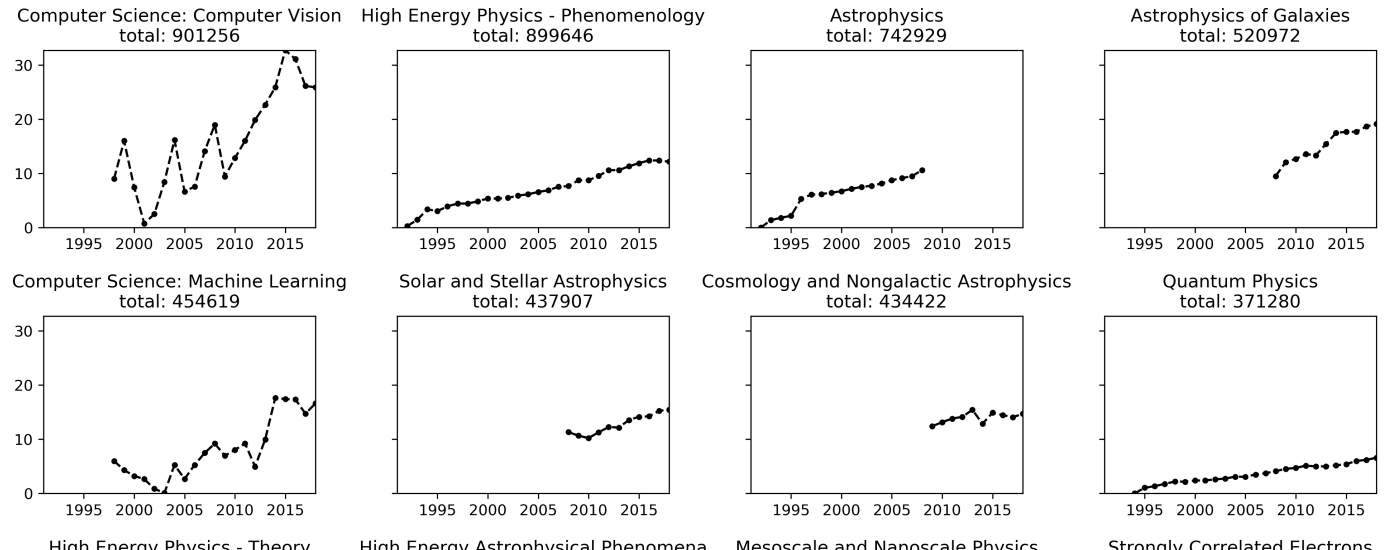

Solar and Stellar Astrophysics

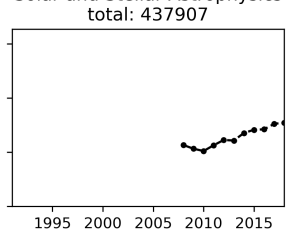

Cosmology and Nongalactic Astrophysics
total: 434422

Quantum Physics

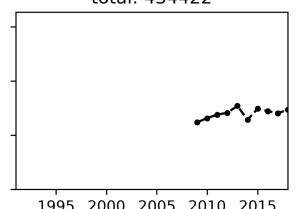

total: 371280

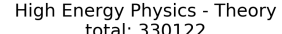

High Energy Astrophysical Phenomena

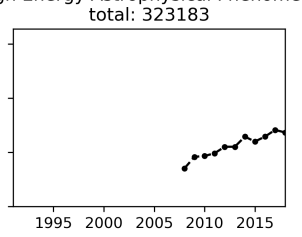

Mesoscale and Nanoscale Physics
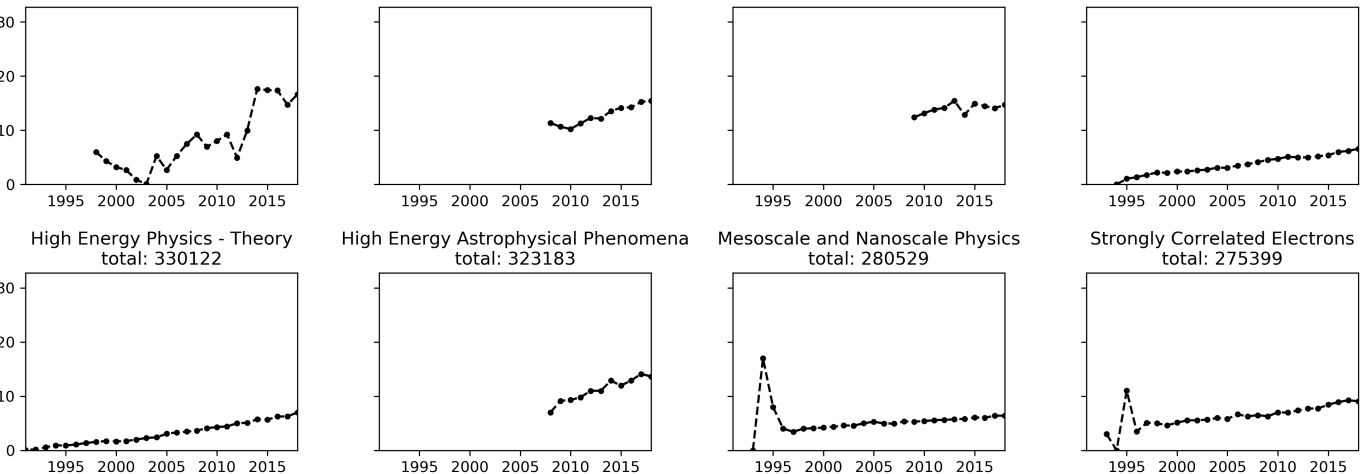

Strongly Correlated Electrons

thematics: Numerical Analysis

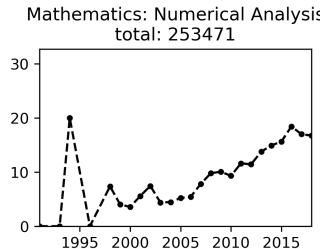

High Energy Physics - Experiment

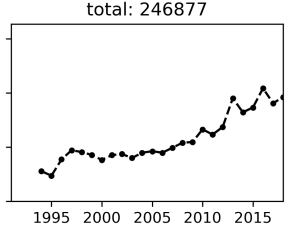

eneral Relativity
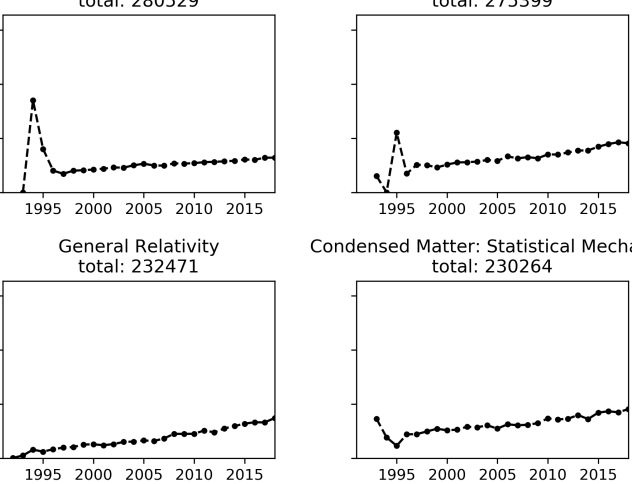

Condensed Matter: Statistical Mechanics

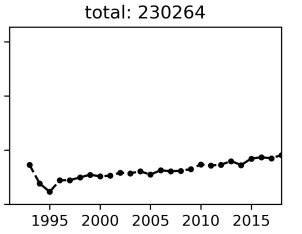

Figure 5: Average number of images per article by year in each category. Ordered by total images in a category, largest to smallest, top-left to bottom right. Top-16 categories only shown here. Total number of images in category indicated under category subtitle.

ways in which image-related and based work have begun to feature more prominently in tasks such as classification, recognition, and analytics, part of the suite of computational and data operations that now permeate and define fields such as computer vision. There is also an upwards trend in categories such as astroph.GA (Astrophysics of Galaxies), which may also be related to an adoption of similar image processing practices. ${ }^{33}$ The most significant results shown here are large increases in image numbers in cs.CV, cs.LG and stat.ML, especially from 2013 onwards. More work would be required to trace the changes in image types and numbers of images, especially as they relate to ML within non-CS disciplines that may have adopted these practices.

Overall, we gained two major insights from our querying of the arXiv image dataset. First, the formats and metadata associated with these images provide 
an awareness of what other computational processes images are subjected to, where they circulate across platforms and networks and what computational image practices are deployed by different scientific communities. Second, by looking at the quantities of image data in relation to the categories or knowledge domains in which they are submitted for preprint, together with information about formatting and software used to create images, we begin to get a sense of a meshwork that enfolds image production, exchange and circulation. This meshwork underpins the practices of sciences at the level of computational and platform infrastructure. Although not 'located' anywhere in the sense in which we ordinarily understand infrastructure, nonetheless this meshwork supports the increasing prominence of images as 'data' of and for scientific research.

\section{Tendencies of the image dataset}

In order to initially gain a sense of what broader image forms might be populating the arXiv image dataset, we randomly sampled subsets of 144 images from different categories and years. These were then analysed and compared for variations in image types and forms. As with any dataset of this scale, it is difficult and time-consuming to physically look at a large number of samples. This presents an interesting challenge to an investigation of the aesthetic tendencies of such image forms. Our approach has been to look at a number of randomly sampled images from across the whole dataset and from category subsets of cs.CV, stat.ML, and cs.AI. These latter were chosen since preprints submitted to these categories use statistical computational imaging techniques such as machine and deep learning.

The images in figure 6 demonstrate a wide variety of image formats and styles. While charts and graphs dominate, there is much diversity within these forms. For example, there are a number of bar graphs that use different colours, spatial arrangements, borders and layouts (such as the placement of a key). Scatterplots similarly take many different forms. There are also a number of heatmaps that stand out in their use of strong gradations of intense colours. We broadly 


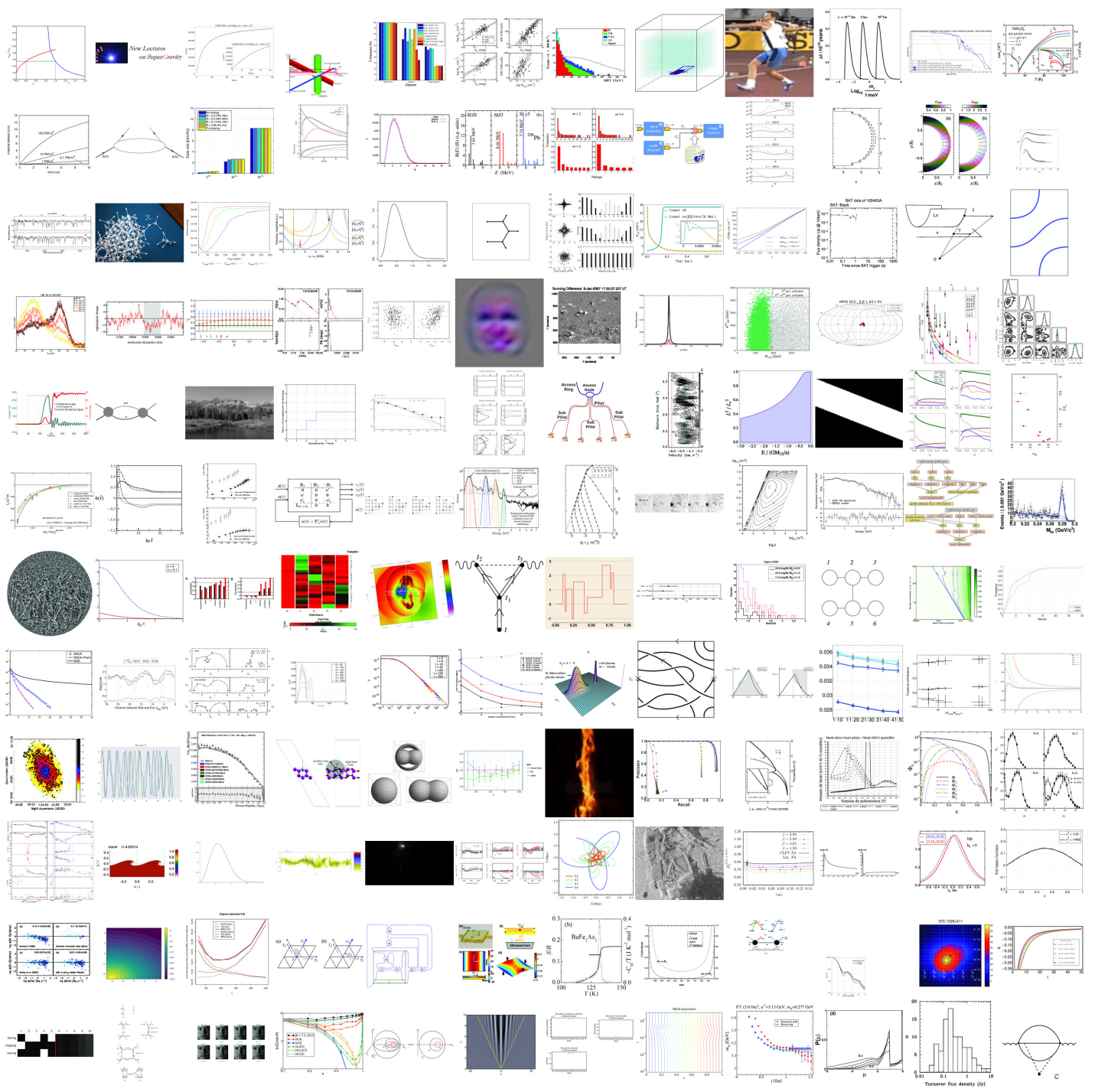

Figure 6: Montage of 144 images sampled randomly from the entire arXiv image dataset, images have been resized to fit within a $240 \times 240$ pixel square. Here we see a diverse collection of images. Image credits.

labelled these kinds of images: diagrams, thinking here of their demonstrative or illustrative functions. There are, on the other hand, several images captured with sensors. Some images appear to be computer-generated simulations of 3D objects or spatial data, as well as schematics and diagrams of technological apparatuses. A number of images composed of symbols and lines appear in this sample that could be describing experimental conditions or presenting mathematical formulas. There are also artifacts of ML computer vision processes, potentially produced at a particular neural network layer or perhaps vectors used 
for facial recognition, e.g. as found in eigenfaces. ${ }^{34}$

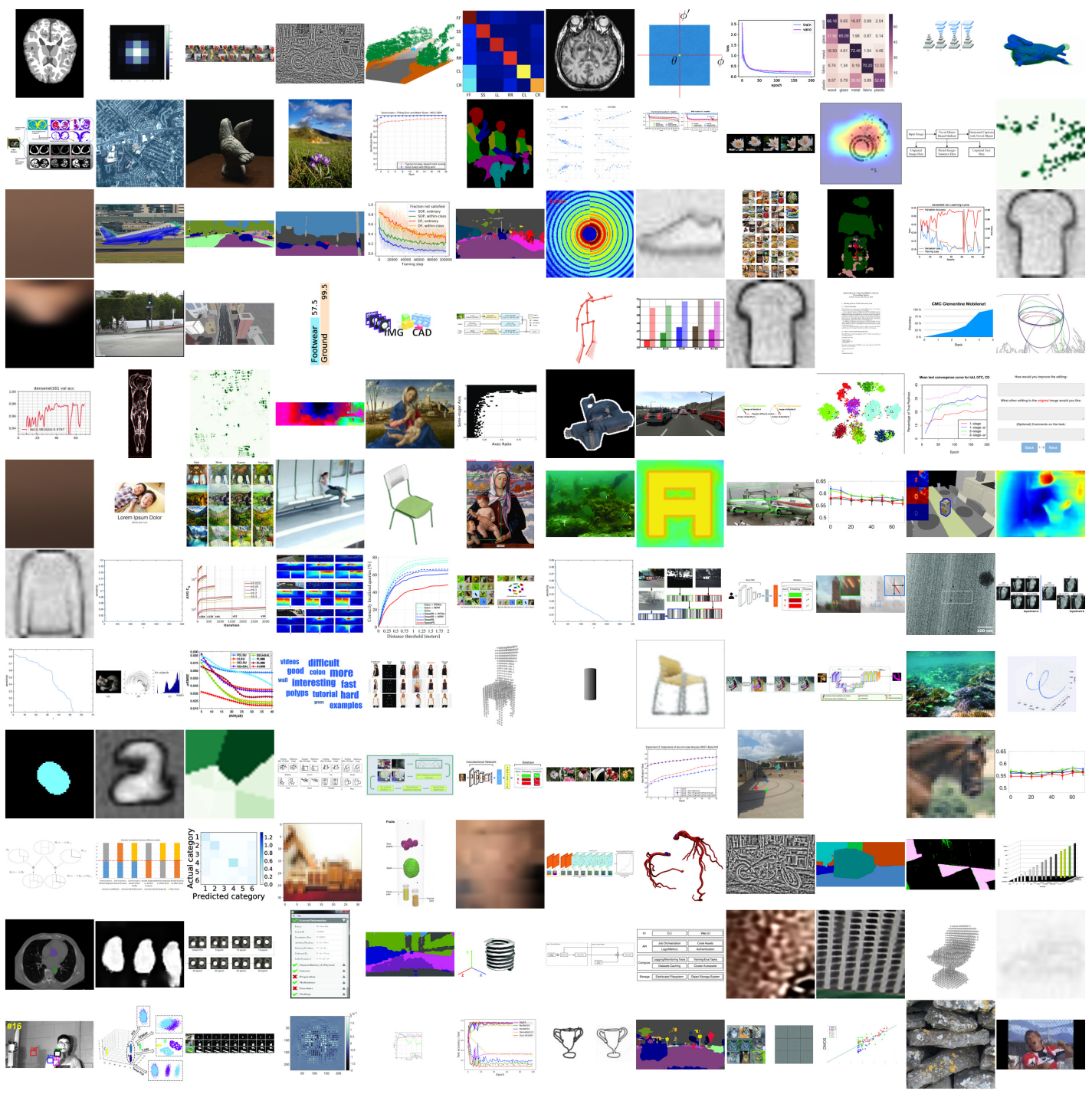

Figure 7: Montage of 144 images taken from October 2018 with a primary category of cs. CV. Image credits.

The images sampled from the primary category of cs.CV (Computer Science: Computer Vision, figure 7) display a different distribution of image forms. ${ }^{35}$ Here we see many more photographic images, images of drawings or artworks, medical imaging, maps, satellite imagery, and segmentation maps. A number of the images appear to have been processed for edge detection, quality reduction, or have overlays added. There are a number of diagrams (graphs, charts, plots, and heatmaps) although far less than in the random sample seen in figure 6. Many of the images here appear to show images found in common ML 
datasets such as MNIST/Fashion MNIST. ${ }^{36}$ Images from MNIST show handwritten digits, while Fashion MNIST shows garments, both in low resolution, grayscale images. In this sample there is a handwritten number " 2 ", and images that appear to show sweaters, shirts, and shoes. These images are likely to have had some additional computer vision processing applied. ${ }^{37}$ Through manually labelling a training dataset and then using the trained classifier, we estimate that approximately $50 \%$ of the images in this subset from cs.CV could be conceived of as a diagram, compared with $91.9 \%$ across the entire dataset (we detail this further below). Overall the images appearing in this sample seem to indicate visual practices specific to computer vision knowledge production, where source images are data inputs; and to its modes of knowledge communication, where image overlays are demonstrative of something being evidenced.

The sample in figure 8 from stat.ML shows diagrams to comprise approximately $80 \%$ of image types. The whole sample set features similar forms as cs.CV including graphs and charts, as well as photographic images with overlays. A number of images appear as multiples, such as the images of traffic intersections, which we have observed occurring across subject categories such as cs.CV and stat.ML. Compared to the cs.CV sample, there is a much higher proportion of diagrams and these appear to have more variation in their forms - bar graph, plot, flow chart, heatmap, tree diagram, scatterplot, $3 \mathrm{~d}$ plot and so forth. The montage of images from cs.AI in figure 9 shows an even higher proportion of diagram images. From labelling each image in the montage manually and calculating totals, diagrams make up $\sim 90 \%$ of the total images. There are only a few images that may have involved a (photographic) sensor. The diagrams are scatterplots, geometric schematics, bar graphs, heatmaps, 3d plots, flow charts and logic diagrams. In cs.AI, diagrammatic images could indicate the gathering of data into visually demonstrative forms; potentially suggesting a propensity to visually explain or communicate AI architectures.

The majority of images across the dataset as a whole could be considered as some sort of diagram; that is, a set of lines that draw parts, whole, elements, and relations as processes, systems, apparatuses or other assemblages. Within 


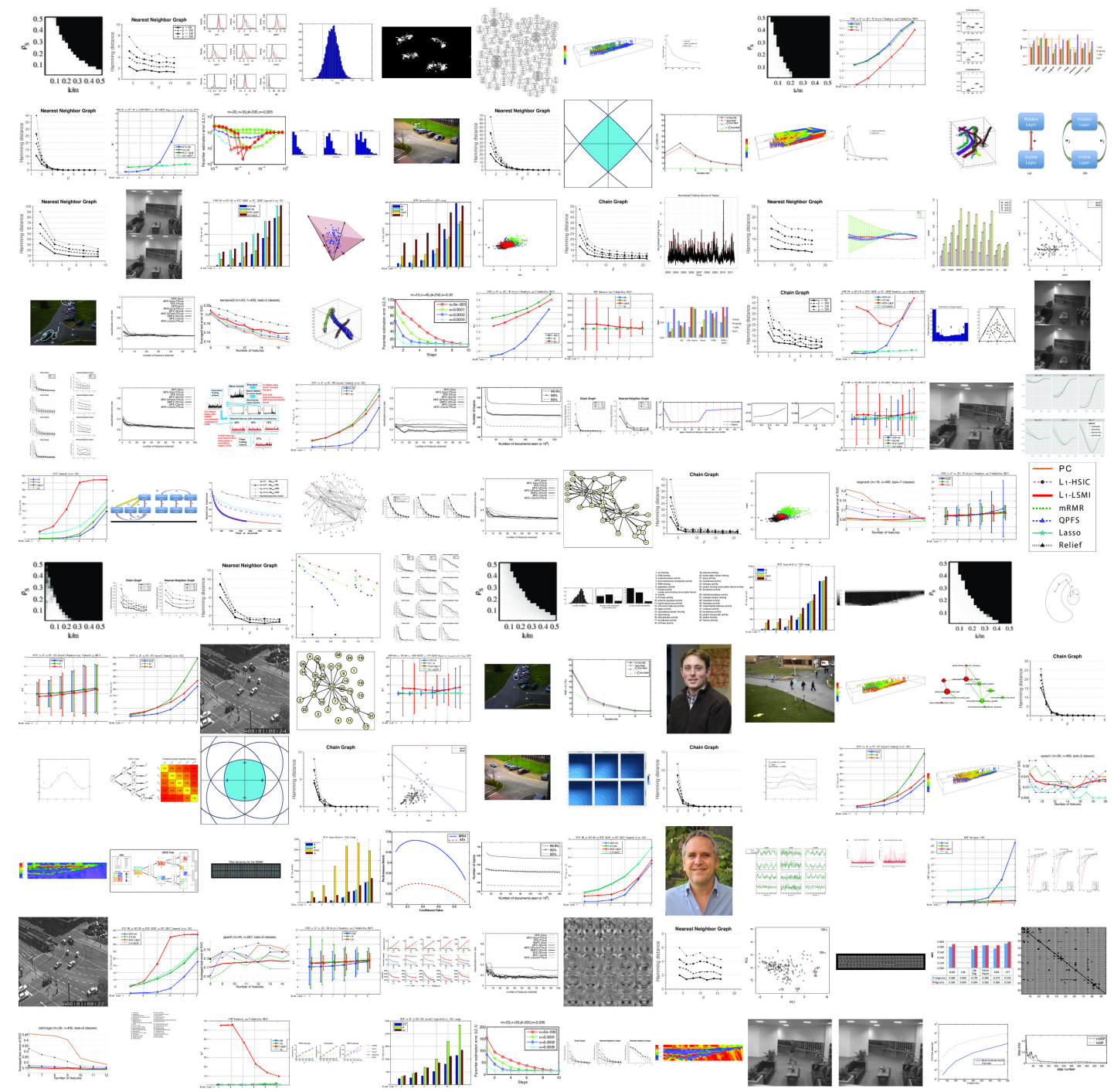

Figure 8: Montage of 144 images taken from October 2012 with a primary category of stat.ML. Image credits.

the diagram subset, there are a number of forms that stand out such as graphs, charts, scatterplots and heatmaps. Many of these forms gather and compress experimental measurements or observations into a graphic form whose contours, spatial relations, labelling, and color indicate trends, correlations, groupings, or patterns. Drawing on other broadly pragmatic uses of the term from scientific literature we bring these under the umbrella of 'diagram': "Diagrams are graphic representations used to explain the relationships and connections between the parts they illustrate." ${ }^{38}$ There is a smaller percentage of images within the dataset that appear to have been captured by a sensor such as a cam- 


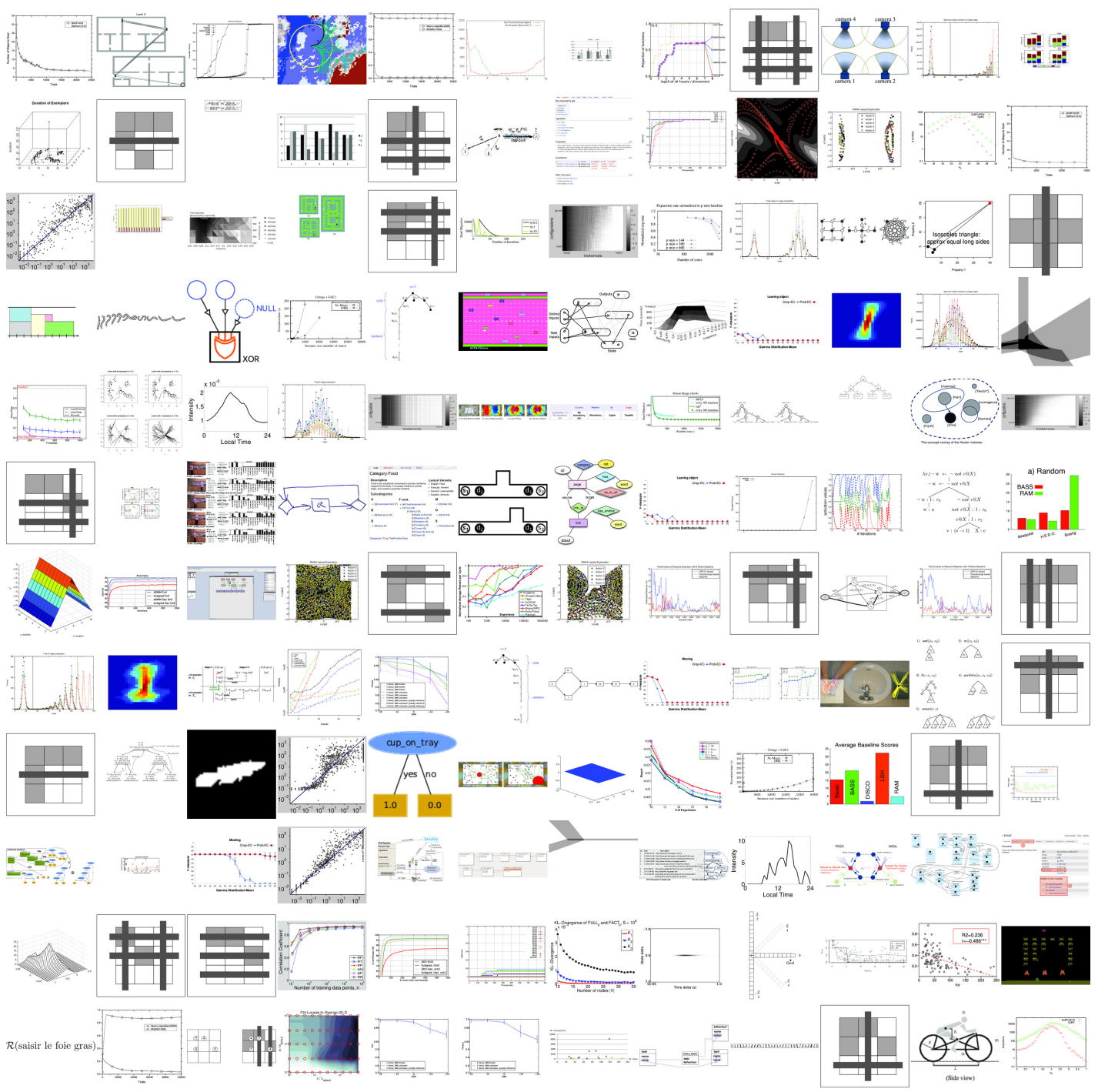

Figure 9: Montage of 144 images taken from 2012 with a primary category of cs.AI. Image credits.

era. These include photographs or other kinds of sensor-produced images but also include overlays such as bounding boxes or motion arrows. A number of images within the dataset could be considered as mathematical formulae, primarily composed of symbols and equations. We assume that these appear where the authors did not choose to use TeX/LaTeX for their typesetting but instead created the images and inserted them into their documents separately. Finally, there are images such as 3D renderings, or images that are composite overlays of part-sensor and part-diagram that do not fall into either the diagram or sensorbased type. We call such images 'mixed.' For most subject categories in the 
arXiv, there are a large proportion of diagrams and a much smaller proportion of images that may have been produced by sensors and mathematical figures as we indicate in figures 6 to 9 .

To estimate the ratios of these different types of images, we trained a neural network classifier that would predict whether an image most closely resembled a 'diagram,' 'sensor' or 'mixed.' 39 To build a training dataset, we manually labelled 9748 randomly sampled images. The decision to create a ternary classifier with a 'mixed' category was intended to capture and highlight the varied operations being performed by images across different kinds of knowledge domains. For example, in our initial querying of image distribution in the dataset, we noticed clusters of images that appeared to be somehow sensor-generated yet re-organised through graphic overlays that seemed to suggest they were being set within a diagrammatic schema. For our purposes, then, a ternary labelling and classification of the images allowed us to view the shifting ratios of image types across arXiv. Our training set produced: 8649 (88.7\%) images as 'diagram,' 477 (4.89\%) as 'sensor' and $622(6.38 \%)$ images as 'mixed.'

We then trained a VGG-16 classifier using the labelled data. ${ }^{40}$ This model was then used on new data inputs in order to give a rough estimation of the changing distribution of sensor-diagram images. Our ternary classifier achieves an accuracy of $92.07 \%$ on the cross-validation dataset. On unseen samples, the classifier predicts $91.9 \%$ of images to be diagrams, $2.1 \%$ of images to be sensor, and $6.0 \%$ to be mixed. This indicates that the classifier is getting similar results to our HIT labels, but the reduction in sensor images in a label with limited data may also point to overfitting the training data, with an increased tendency to predict either diagram or mixed. This process of classification was used to inform our choices of arXiv categories to look at more deeply and to query the features of images across time in specific fields of scientific research. Since this is all highly dependent on the labelling process, random image sampling, and training of the network, these results (shown in figure 10) are only a preliminary guide for further research and inquiry. 

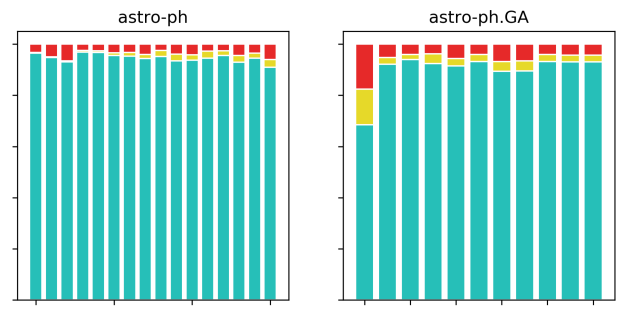

Cs.Al

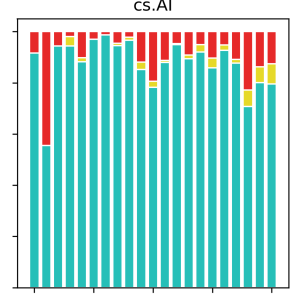

cs.LG

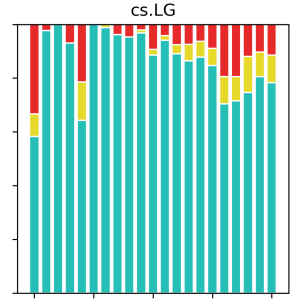

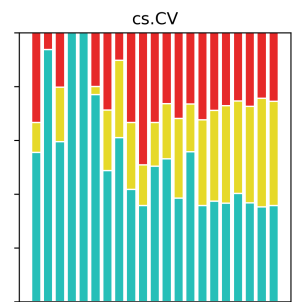

math-ph

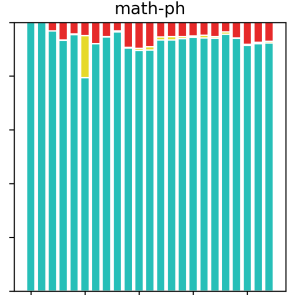

astro-ph.IM

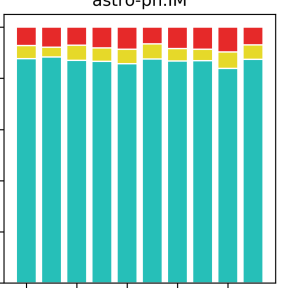

cs.GR

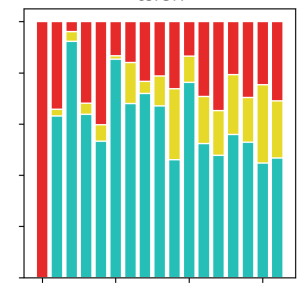

nlin.CG

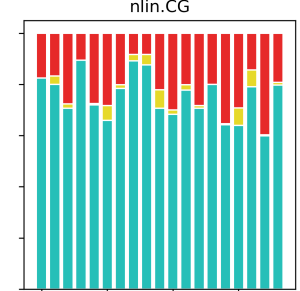

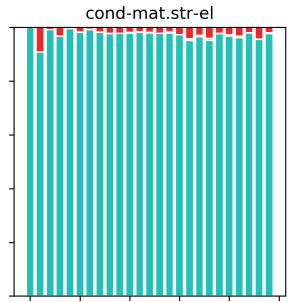

Cs.IT

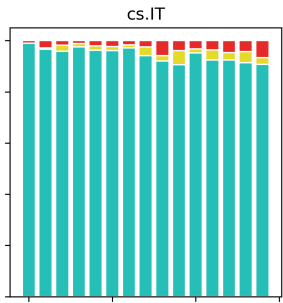

stat.ML

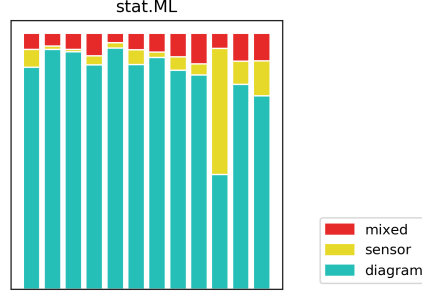

Figure 10: Ratio of diagram/sensor/mixed image classifications predicted using custom ternary classifier. Maximum of 2000 images sampled from any given category-year combination. Categories shown are hand selected. Image with all categories.

Interestingly for our concerns, the category of cs.CV shows an increasing trend in the last decade away from diagram-type images towards an almost even threeway split between diagram, sensor, and mixed, as seen in Figure 10. As such, cs.CV is moving towards a much higher ratio of sensor-based images than comparative categories. The stat.ML category exhibits a similar trend, although not to the same degree. Categories such as astro-ph and related astrophysics fields are predicted to contain a majority of diagram images, remaining consistent across the time period sampled. Categories such as nlin.CG (Physics: Cellular Automata and Lattice Gases) and cs.GR (Computer Science: Graphics) are predicted as containing a high proportion of 'mixed' images. The aforementioned cs.CV category is predicted as having the highest proportion of 'sensor' images, unsurprising given the content and source material that computer vision often works with.

Due to the enormous number of images, we chose to limit our initial inquiry to 
only a few categories. Here, we specifically focus on looking at images from cs.CV (Computer Science: Computer Vision and Pattern Recognition), cs.AI (Computer Science: Artificial Intelligence), and stat.ML (Statistics: Machine Learning). ${ }^{41}$ We expected that cs.CV and stat.ML would have increased numbers of images in the time following significant ML, deep learning, and image processing developments such as ILSVRC 2012, when a deep neural network was used to significantly improve upon previous results. ${ }^{42}$ The initial metadata statistics and image form analysis confirms this and shows that there is an increase in images being submitted in preprints in these categories. Additionally, there is a shift away from the use of diagrams in the preprints in these categories and towards other image types (both sensor-based and 'mixed' images) becoming more prevalent.

The montages and classifier results give us a sense of the quantities, types and proportions of images/types we might find in specific categories of arXiv images, but they do not show us anything further about the relations of these images to each other or their circulation throughout the corpus of research that comprises arXiv. We wanted to get a sense of how images, whether diagrams, sensor-based or hybrids of the two, move in and out of proximity and/or distance to each other. We aimed also to explore preoccupations with a particular type of image within a category or domain of knowledge production. Querying and providing a 2-D mapping of the ML methods we have used to think about the images of arXiv shows the distribution of such images according to categories - or domains of knowledge production in the sciences - via relations of image-centric proximity to and distance from each other. A classifier is used in data science to group members into separate classes in which the class denotes a consistent similarity of some particular thing. We have used a form of supervised learning in which we initially trained our classifier according to a set of 3 classes that were manually labelled: that is, the classes of images belonging to 'diagram', 'sensor-based' or 'mixed'. Once trained the classifier was then run over the publication submission history of particular arXiv categories. Another ML method for looking at spatial distribution of similarity and differences via 
clustering of data is the t-SNE algorithm. t-SNE does this by performing dimensionality reduction and placing all images in a 2-dimensional spatial mapping, which can then be analysed visually by human eyes.

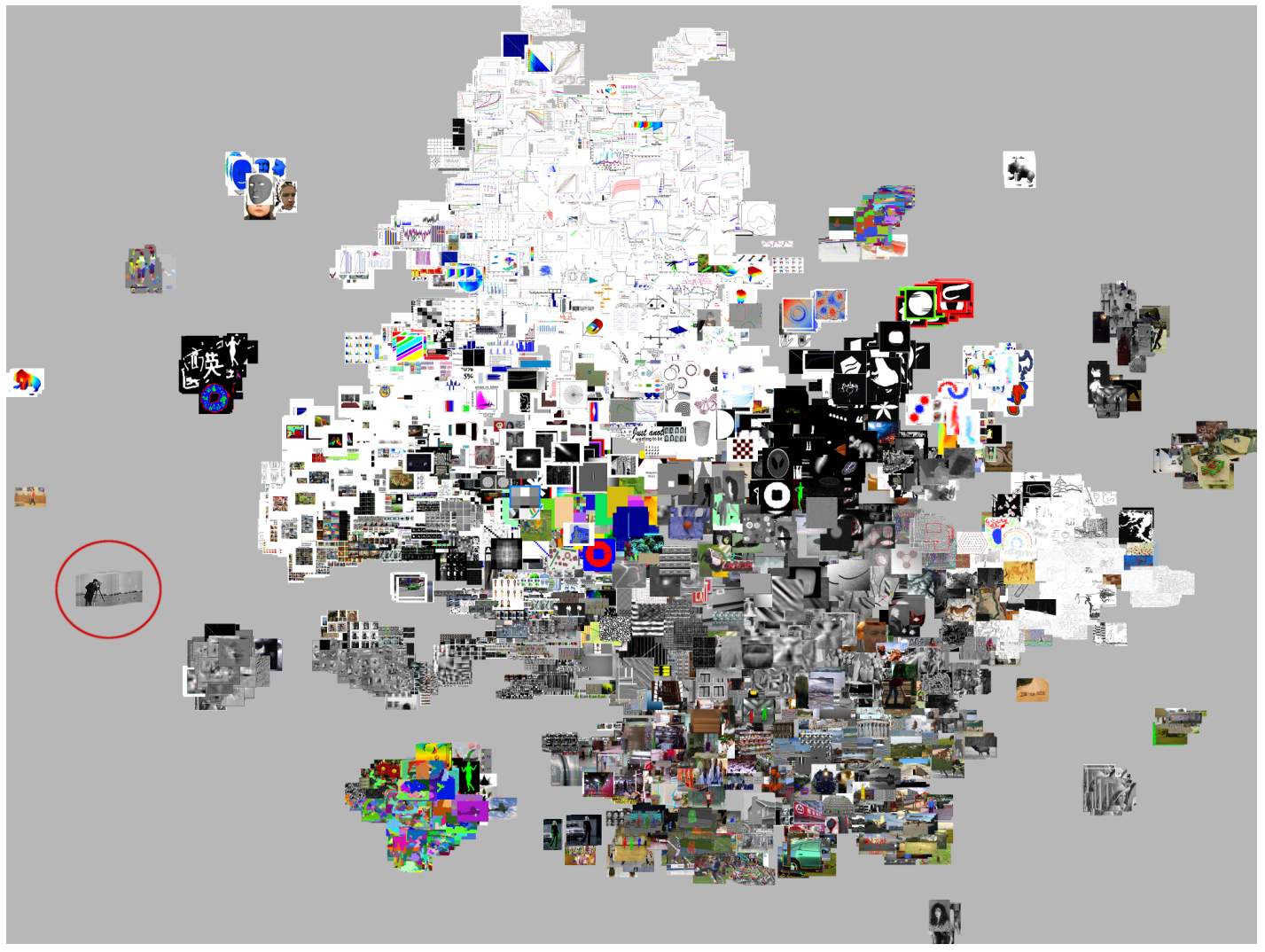

Figure 11: Visualisation of t-SNE dimensionality reduction run on 8381 images from cs.CV from 2012. Shows distribution of images according to the ML process. Grouping of the same “cameraman” image circled in red. Image credits.

We ran a t-SNE algorithm across various subsets of images in order to look for patterns of similarity, without any explicit reference to disciplinary boundaries. ${ }^{43}$ We took a VGG-16 CNN model, pre-trained on ImageNet and used the second last fully connected layer to obtain image features. ${ }^{44}$ This provides a 4096-dimension feature vector for each image - much smaller than the original pixel data but embedded with specific features of the classifier network. Principle component analysis, a standard process in ML dimensionality reduction, is then applied to this feature vector to reduce the size of the vector and remove redundancy. This allows us to convert the 4096-dimension vector to 300 dimensions, while retaining almost all of the variance. t-SNE is then used to find 
two-dimensional coordinates for each of these image vectors, which can then be used to plot the original images in 2-dimensional space. It does this by iteratively calculating the nearest neighbours for each data point (each image) and reorganising the two dimensional output until the data is placed optimally. ${ }^{45}$ The results of this process create vectorised observations drawn from the features of the images such as textures, colours, and contours.

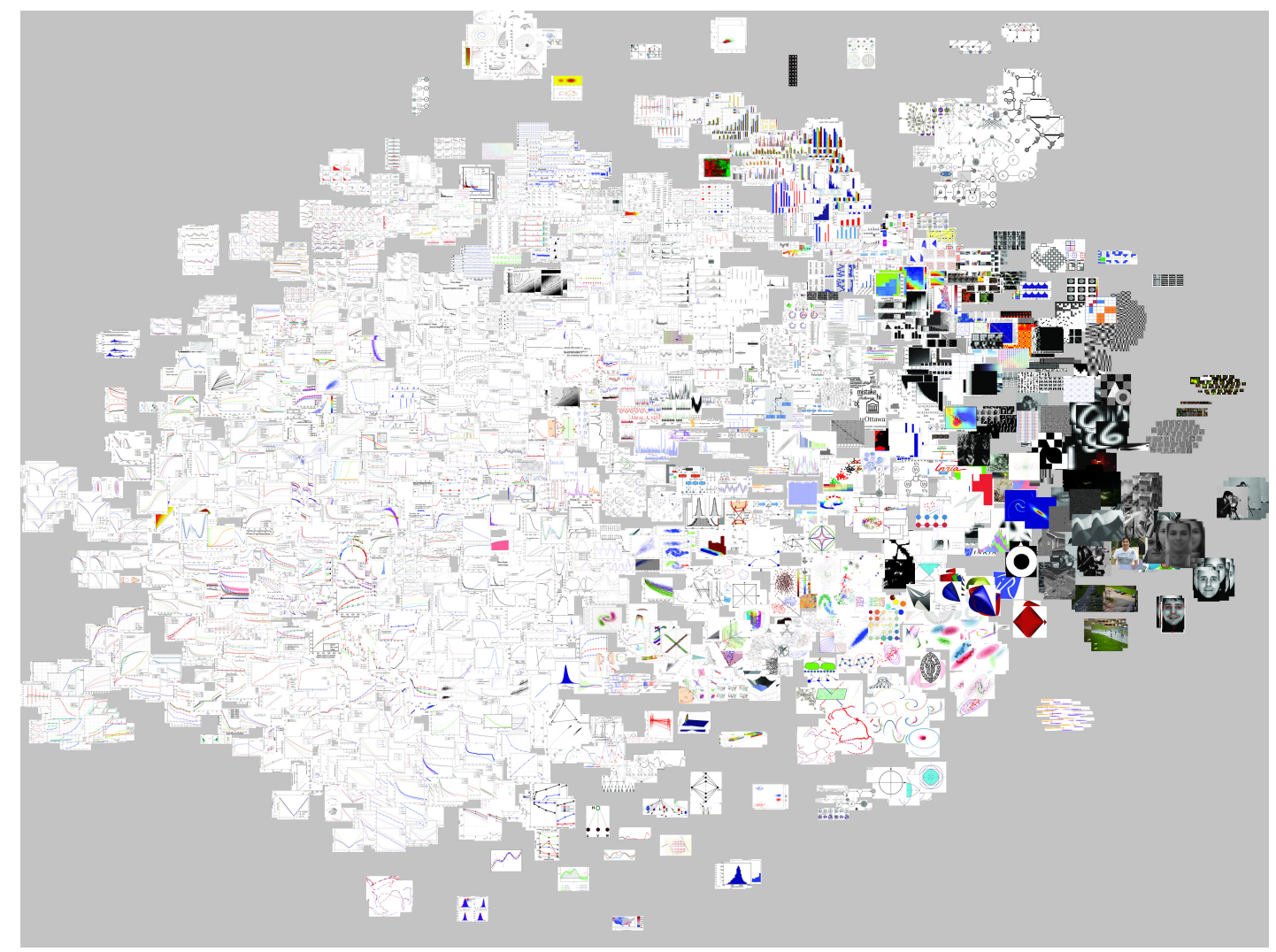

Figure 12: Visualisation of t-SNE dimensionality reduction run on 2889 images with a primary category of stat.ML from 2012. Image credits.

We can glean the distribution of image forms from the proximity and clustering of images across the particular categories/years/formats being queried. In the t-SNE mapping of cs.CV images in figure 11 , there are small separate peripheral image clusters that seem to group according to colour content or shapes within the image. Images with white backgrounds such as graphs and charts appear mainly in the upper half of the distribution, separated from the predominantly greyscale images that fall mainly across the lower half. A cluster of 
the same photograph of a man looking through a camera (a public-domain test image often used in MATLAB, titled "cameraman"46) appears at the centre of the left side as a distinct group (circled in figure 11). Two main vectors dominate the distribution of images here: the separation of some images into distinct groupings (or clusters) in which repeated image content or repeated dominant features are found; and the overall organisation of the larger group of images according to colour content. Here the central cluster moves top down from predominantly white background images, to some distinct colours, to images that are largely gray, and then finally to images that are mostly muted colours. This also coincides with our quantitative findings on ratios of image types within stat.ML, shown in figure 10. In 2012, this category had a much higher proportion of diagram-type images, appearing in the t-SNE visualisation as the large spread of white-background images on the entire left-hand side. There is a much smaller proportion of photographic images, towards the centre-right, including the "cameraman" image at the far right edge.

The clustering found in the t-SNE images shows that features acquired through the VGG-16 pre trained classifier are working to seriate images along specific vectors. Here larger image types that we have classified as diagrams, sensorbased, and hybridised diagram-sensors (or 'mixed') drive a macro-observation of such vectors. But zooming in on, for example, the stat.ML t-SNE and focusing on the diagram images (see figure 12), we can see other kinds of distribution relations emerging between the diagram forms themselves. In the figure 13 detail, features such as slope of lines, repetition and comparative repetition, small multiples, and colour have a generative effect on clustering. For example, the grouping of images in the top-right of this detail mostly involves small multiples of comparative graphs, while the images towards the centre-left mostly show logarithmic curves. But also interestingly, the further one zooms in to the t-SNE, the more the distinctiveness of clustering as definitive diagram form falls off. Instead all the clusters tend toward all other clusters in their proximity. A patterning of diagram relations at the level of variable similarities emerges. From this, we propose that the ML and deep learning techniques we use func- 


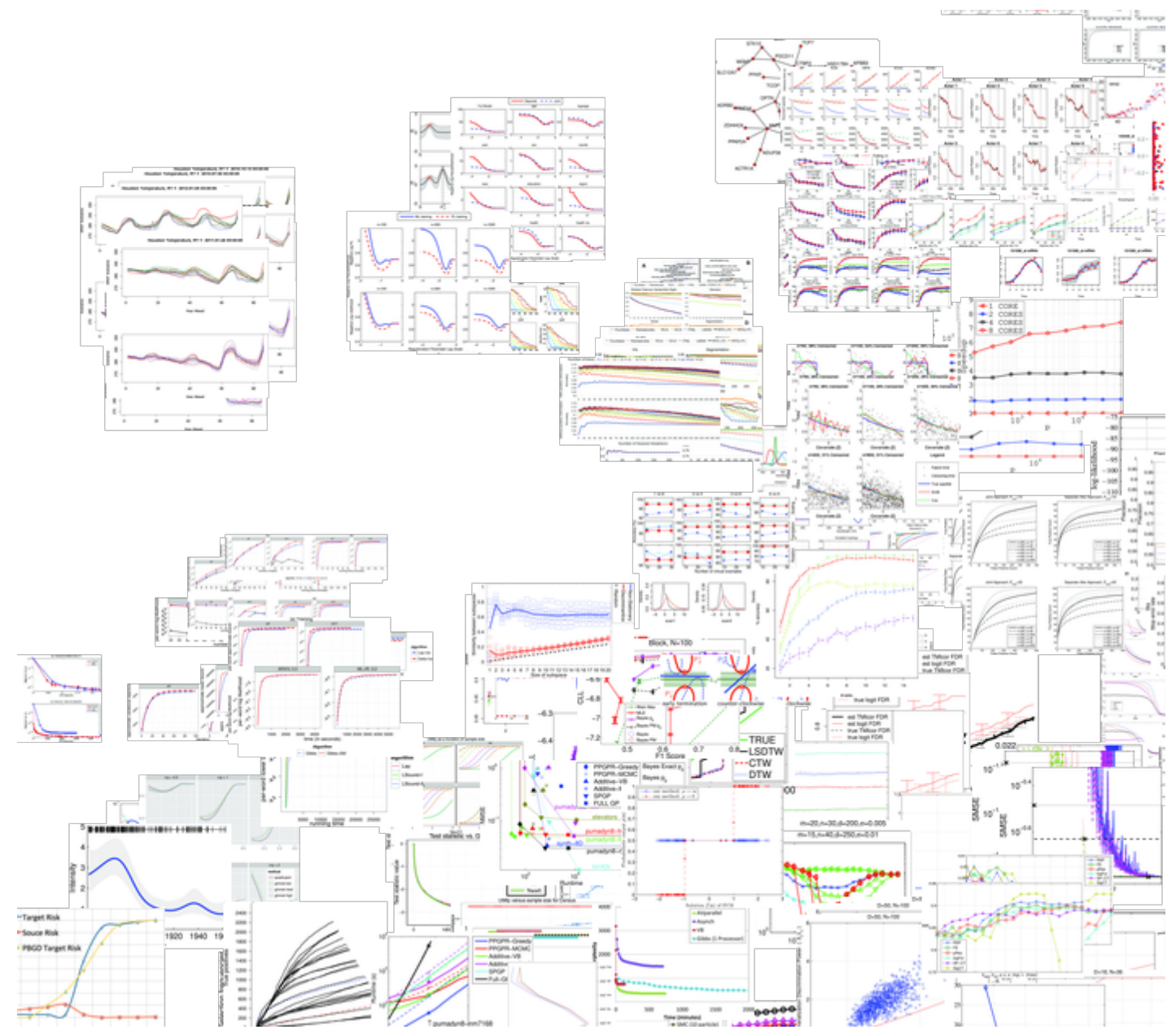

Figure 13: Detail of stat.ML 2012 t-SNE shown in figure 12 .

tion to observe a transversal plane in the image arXiv dataset. Moving across this transversal plane affords a different way of seeing image relations, tendencies and potentially their associations with scientific communities of practice. $\mathrm{t}$-SNE and VGG processes traverse the plane without predicating any representational or indexical function. Yet they do more than act as simple quantitative segmentations of the image data. They offer us tendencies that images as forms might be following in relation to a large corpus of scientific research and how such forms might be moving in relation to each other and the epistemological endeavours in which they are engaged. 


\section{Conclusion}

Exploratory approaches to large image datasets can be of interest to data scientists and to transdisciplinary researchers in the postdigital humanities, software studies, and critical AI studies. They are especially resonant in contextualising the trans-contextual flows of changing visual techniques as they percolate across scientific fields and subfields. It is somewhat ironic that in aggregating a large image dataset, that incorporates images from the very sciences most focused on their labelling and recognition, we discover the lively 'resistance' of such images to classification. This 'resistance' lies not simply at the level of size and format - typically understood as data cleaning problems - but at the interlacing of image types found in preprint publishable scientific research. One lesson of the arXiv image dataset, a dataset rooted in epistemically privileged contemporary sciences such as physics, computer science, mathematics, and statistics, is that categories do not necessarily map onto image form; indeed they might mask the meshwork of images. The endemic diagram-type images in arXiv confounds current database ontologies that are maximised for specific ML and deep learning tasks such as face and object recognition, making 'scientific images' in their heterogeneity themselves largely unclassifiable with current tools. Our explorations have led us someway down a path toward a rough ternary mode of classification of images of arXiv. But we have also discovered that there seems to be no standard statistical method for segmenting their image 'forms' because the forms present continuous variation across classificatory boundaries. If we have asked why no attention has been paid to such images then our explorations demonstrate that more attention is due.

In making image formatting a point of interest in our exploration of the arXiv image dataset metadata, it has been possible to speculate about the relation of formatting to other recent trends in the relations of images to little remarked visual practices of ML and computation more generally: web-scraping for data collection and increased cross-platform circulation of images particularly stand out. Ongoing research in this project will continue to link aspects of the meta- 
data such as the 'creator' information to keyword searches performed on the image captions. We hope this might render some relations between the mode of generating images (programmatically or manually, for instance) and images that are direct outputs of ML-oriented research. This, we suspect, may begin to circumscribe what ML as a research practice 'looks like.'

An empirically exploratory approach to large (scientific) image datasets is not context-free, but addresses the challenge of crafting ways to engage with the circulatory visual cultures of the sciences, especially those that are ML-focussed or associated. Our research and approach is interested as much in the dataset relations ML techniques reconfigure as the 'imagistic' nature of contemporary science image data. Our 'experiments' in assembling a large image dataset and our visual/ML observations of it have been recursive, often involving a return to running a new process on earlier quantitative findings. This has allowed us to remain 'open' to the dataset itself, while acknowledging that each return is also a computational incursion into and reconfiguration of the image data itself. This is not so unlike any work on large datasets and the building of ML or deep learning models. Data work today involves: running many iterations or 'epochs'; constant tweaks and optimisations of data and parameters; and the inevitable discovery that predictions expose over and underfitting. We suggest that an experimental embrace of this recursivity might also facilitate knowledge of another kind of visuality for contemporary scientific images, that takes into account their immanent circulatory relations and the technical infrastructures and operation through which they circulate.

\section{Acknowledgments and disclosure of funding}

The authors thank the reviewers for their valuable feedback. The full title of this project is: 'Re-imaging the Empirical: statistical visualisation in art and science' (2017-2021). Professor Anna Munster (University of New South Wales) is lead investigator and collaborating with Professor Adrian Mackenzie (Australian National University) and Dr Kynan Tan, Postdoctoral Fellow (University of New 


\section{South Wales). This is a Discovery Project funded by the Australian Research Council.}

\section{Notes}

${ }^{1}$ Further information about the broader project, especially concerning our aims and methods, is available at: https://github.com/re-imaging/re-imaging/wiki.

${ }^{2}$ Michael Friendly, “A Brief History of Data Visualization,” in Handbook of Data Visualization, ed. Chun-houh Chen, Wolfgang Karl Härdle, and Antony Unwin (Springer Science \& Business Media, 2007), 42ff.

${ }^{3}$ Janet Vertesi, Seeing Like a Rover: How Robots, Teams, and Images Craft Knowledge of Mars (Chicago, IL and London: University of Chicago Press, 2015); Catelijne Coopmans et al., Representation in Scientific Practice Revisited (Cambridge, MA: MIT Press, 2014); Lorrraine Daston and Peter Galison, Objectivity (Brooklyn, NY: Zone Books, 2007).

${ }^{4}$ Christopher Andreas Clark and Santosh Kumar Divvala, "Looking Beyond Text: Extracting Figures, Tables and Captions from Computer Science Papers" (2015), 2.

${ }^{5}$ Figures, such as plots, graphs and charts, are "used to display trends and patterns of relationship, but they can also be used to communicate processes or display complicated data simply." The Writing Centre UNC, "Figures and Charts," 2020, University of North Carolina, accessed June 25, 2020, https://writingcenter.unc.edu/tips-andtools/figures-and-charts/. See also, Scott Mogull and Candice Stanfield-Wiswell, "Current Use of Visuals in Scientific Communication," Proceedings of the IEEE, July 2015, 1-6, https://doi.org/10.1109/IPCC.2015.7235818.

${ }^{6}$ Olga Russakovsky et al., "ImageNet Large Scale Visual Recognition Challenge,” International Journal of Computer Vision 115, no. 3 (2015): 211-52, https://doi.org/10.1007/s11263-015-0816-y.

${ }^{7}$ An entire subfield of ML research is now devoted to 'explainability' of the black boxes of, especially, neural networks. Visualisation is used in the form of diagrams, gifs, videos and interactive interfaces that attempt to demonstrate what is happening at the computational and statistical level of a model's operations. See for example, Giuseppe Casalicchio, Christoph Molnar, and Bernd Bischl, "Visualizing the Feature Importance for Black Box Models," in Machine Learning and Knowledge Discovery in Databases, ed. Michele Berlingerio et al. (Cham: Springer International Publishing, 2019), 655-70.

${ }^{8}$ Bruno Latour, "Visualisation and Cognition: Drawing Things Together," in Knowledge and Society: Studies in the Sociology of Culture Past and Present, ed. H. Kuklick, vol. 6 (Greenwich, CT: Jai Press, 1986), 1-40; Bruno Latour and Steve Woolgar, Laboratory Life: The Construction of Scientific Facts (Princeton, NJ: Princeton University Press, 1986), 45ff.

${ }^{9}$ The diverse contributions that make up the anthology Representation in Scientific Practice Revisited are indicative of this doubled performativity of inscriptions. Coopmans et al., Representation in Scientific Practice Revisited. 
${ }^{10}$ The early approach to STS investigations of the use of visual materials in science was based in an ethnography of specific science laboratories and communities of practice. Some of this important work continues and also tries to contend with the highly distributed infrastructure of scientific practices and communications (for example in Janet Vertesi's work already cited). We are suggesting that in looking at how images circulate across different fields of scientific practice and the mechanisms for such circulation, which include both preprint repositories and image datasets, we need to take into account a different form and mode of circulation. This is no longer the direct work of what images or scientists do but is part of an entire technical and platform infrastructure and set of operations, which surpass the 'traditional' representational work of images or the various ways scientists represent their findings.

${ }^{11}$ James E. Dobson, Critical Digital Humanities: The Search for a Methodology (Champaign, IL: University of Illinois Press, 2019). We also draw on the spirit of Joanna Drucker's approach here, when she makes a case for ambiguity and contradiction as key to graphical methods that are used to visualise data. See Johanna Drucker, "Graphical Approaches to the Digital Humanities," in A New Companion to Digital Humanities (John Wiley \& Sons, Ltd, 2015), 238-50.

${ }^{12}$ See for example, Claus Weihs and Katja Ickstadt, "Data Science: The Impact of Statistics," International Journal of Data Science and Analytics 6, no. 3 (2018): 189-94, https://doi.org/10.1007/s41060-018-0102-5.

${ }^{13}$ arXiv is owned and operated by Cornell University as an online open access repository. "arXiv.Org e-Print Archive," 2020, accessed October 16, 2019, https://arxiv.org/.

${ }^{14}$ Colin B. Clement et al., "On the Use of ArXiv as a Dataset," April 30, 2019, 1, arXiv: 1905.00075. We also acknowledge that there are other preprint repositories that act to disseminate scientific research and debate through online platforms. One example is bioRxiv, accessible at https://www.biorxiv.org/. However, arXiv is the largest open access repository which also provides access to the repository itself as a bulk data download. It was this latter aspect that made it suitable to our research interests.

${ }^{15}$ Alessandro Delfanti, "Beams of Particles and Papers: How Digital Preprint Archives Shape Authorship and Credit," Social Studies of Science 46, no. 4 (2016): 629, https://doi.org/10.1177/0306312716659373.

${ }^{16}$ For statistics and analysis provided by arXiv.org, see "arXiv Submission Rate Statistics," 2020, accessed March 13, 2020, https://arxiv.org/help/stats/2018_by_area/index.

${ }^{17}$ Sergio Sismondo, "Sorting on arXiv: Introduction to an Ad Hoc Section:" Social Studies of Science, August 23, 2016, https:// doi.org/10.1177/0306312716661429; Luis Reyes-Galindo, "Automating the Horae: BoundaryWork in the Age of Computers:" Social Studies of Science 46, no. 4 (2016): 586-606, https://doi.org/10.1177/ 0306312716642317; Kristrún Gunnarsdóttir, "Scientific Journal Publications: On the Role of Electronic Preprint Exchange in the Distribution of Scientific Literature," Social Studies of Science, June 29, 2016, https://doi.org/10. $1177 / 0306312705052358$.

18“arXiv Bulk Data Access,” 2019, accessed July 10, 2020, https://arxiv.org/help/bulk_data.

${ }^{19} \mathrm{TeX} / \mathrm{LaTeX}$ is a document preparation and typesetting system that is widely used in science and mathematics to format papers. See "LaTeX - A Document Preparation System," 2020, accessed July 16, 2020, https://www.latexproject.org/. 
${ }^{20}$ For examples, see Clement et al., "On the Use of ArXiv as a Dataset"; Alexander A. Alemi and Paul Ginsparg, “Text Segmentation Based on Semantic Word Embeddings," March 18, 2015, arXiv: 1503.05543; Michał Łopuszyński and Łukasz Bolikowski, "Tagging Scientific Publications Using Wikipedia and Natural Language Processing Tools. Comparison on the ArXiv Dataset," 2014, 16-27, arXiv: 1309.0326; and Stefan Pohl, "Using Access Data for Paper Recommendations on ArXiv.Org," April 23, 2007, accessed October 10, 2019, arXiv: 0704.2963, http://arxiv.org/abs/0704.2963.

${ }^{21}$ Siegel et al. use the ${ }^{\mathrm{AT}} \mathrm{T} \mathrm{X}$ code describing image placements found in the arXiv bulk data download for detecting image placement. Noah Siegel et al., "Extracting Scientific Figures with Distantly Supervised Neural Networks," Proceedings of the 18th ACM/IEEE on Joint Conference on Digital Libraries - JCDL '18, 2018, 22332 , https://doi.org/10.1145/3197026.3197040. We are not aware of other research investigating the image content of this dataset.

${ }^{22}$ A notable exception is David Ribes, "STS, Meet Data Science, Once Again," Science, Technology, \& Human Values 44, no. 3 (2019): 514-39, https://doi.org/10.1177/0162243918798899.

${ }^{23}$ Edward Tufte on the other hand would argue: "Graphics are instruments for reasoning about quantitative information." Edward R. Tufte, The Visual Display of Quantitative Information, vol. 2 (Cheshire, CT: Graphics press LLC, 2001), 11. Our research suggests that images including graphics play very different roles as they become constituent members of aggregates or collections, for example.

${ }^{24}$ For example, the ImageNet dataset has become the de facto standard for visual recognition challenges, as well as being the primary dataset for training feature extractors. See Russakovsky et al., "ImageNet Large Scale Visual Recognition Challenge"; Pierre Stock and Moustapha Cisse, "ConvNets and ImageNet Beyond Accuracy: Understanding Mistakes and Uncovering Biases," in Lecture Notes in Computer Science (Including Subseries Lecture Notes in Artificial Intelligence and Lecture Notes in Bioinformatics, vol. 11210 (Springer Verlag, 2018), 504-19.

${ }^{25}$ For full technical details, code, and step-by-step instructions for downloading and organising the dataset, please see our github: https://github.com/re-imaging/re-imaging.

${ }^{26}$ Information regarding the downloading of bulk data can be found at https://arxiv.org/help/bulk_data_s3.

${ }^{27}$ The collection was announced in January 2019 by IBM and caused an uproar due to the lack of consent by users who had uploaded facial images to Flickr. Michele Merler et al., "Diversity in Faces," arXiv e-prints, January 2019, arXiv:1901.10436.

${ }^{28}$ Importantly the standardisation is a result of industrial regulation by the International Standards Organisation. 2008 was a key turning point with the introduction of PDF/E (ISO standard 24517) introduced as a special PDF format for technical documents in the fields of engineering, architecture and geo information systems.

${ }^{29}$ Jonathan Sterne, MP3: The Meaning of a Format (Durham, NC and London: Duke University Press, 2012), 51.

${ }^{30}$ The SQLite database file with both metadata and images tables is available to use at https://drive.google. com/open?id=1FISX3DpSQmZr9BFexAei64vuoh6zKNE6 (847 MB compressed tar archive) or with additional 
captions table at https://drive.google.com/file/d/1Gwllzb0Iv5bt9bGY8hrwki6Z8kZTuoNR (1.3 GB).

31 "arXiv Submission Rate Statistics."

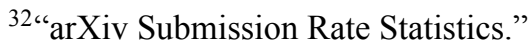

${ }^{33}$ To see the statistics of images per article for all categories, see https://github.com/re-imaging/re-imaging/blob/ master/statistics/stats_images_cat_year.org.

${ }^{34}$ M.A. Turk and A.P. Pentland, "Face Recognition Using Eigenfaces," in 1991 IEEE Computer Society Conference on Computer Vision and Pattern Recognition Proceedings (June 1991), 586-91, https://doi.org/10.1109/ CVPR.1991.139758.

${ }^{35}$ The images in Figure 7 are taken from 2018 as opposed to images in Figures 8 and 9 which are taken from 2012. This was done to select images for cs.CV that articulated the types of images appearing as the number of images per article increased drastically. This also shows the changing ratios of image types in this particular category.

${ }^{36} \mathrm{Fashion}$ MNIST is available at https://github.com/zalandoresearch/fashion-mnist. MNIST can be found at http://yann.lecun.com/exdb/mnist/.

${ }^{37}$ These images come from the same paper, which contained a total of 584 images, see https://arxiv.org/abs 1810.02340 .

${ }^{38}$ Muriah J Umoquit et al., "A Multidisciplinary Systematic Review of the Use of Diagrams as a Means of Collecting Data from Research Subjects: Application, Benefits and Recommendations," BMC Medical Research Methodology 11, no. 1 (2011): 1, https://doi.org/10.1186/1471-2288-11-11.

${ }^{39}$ This work was performed by the three researchers on this project and assigned independently to each as a human intelligence task or HIT. Our decisions are of necessity biased according to our individual and collective approaches to labelling these categories.

40“Keras Documentation: VGG16 and VGG19,” 2020, accessed July 16, 2020, https://keras.io/api/applications/ $\operatorname{vgg} /$.

${ }^{41}$ The cs.LG (Computer Science: Machine Learning) category is also significant to this research, however it is cross-posted to stat.ML and has much in common with cs.CV. Therefore we have not used it as one of our chosen categories in order to cover a greater breadth of ML research.

${ }^{42}$ Alex Krizhevsky, Ilya Sutskever, and Geoffrey E. Hinton, "ImageNet Classification with Deep Convolutional Neural Networks," in Advances in Neural Information Processing Systems 25, ed. F. Pereira et al. (Curran Associates, Inc., 2012), 1097-105.

${ }^{43}$ Laurens van der Maaten and Geoffrey E. Hinton, "Visualizing Data Using T-SNE," Journal of Machine Learning Research 9 (Nov 2008): 2579-605 We used the CUDA accelerated implementation tsne-cuda, see David M. Chan et al., "GPU Accelerated T-Distributed Stochastic Neighbor Embedding," Journal of Parallel and Distributed Computing 131 (2019): 1-13. 
${ }^{44}$ Karen Simonyan and Andrew Zisserman, "Very Deep Convolutional Networks for Large-Scale Image Recognition," September 4, 2014, arXiv: 1409.1556. We used the implementation provided by the Keras library. "Keras Documentation."

${ }^{45}$ Thanks to Machine Learning for Artists (ML4A) for code examples for this process of feature extraction. See "image-tsne.ipynb" in Gene Kogan and James Oldfield, "Machine Learning For Artists," 2019, accessed October 16, 2019, https://github.com/ml4a/ml4a-guides.

46“Public-Domain Test Images for Homeworks and Projects," 2020, accessed October 16, 2019, https://homep ages.cae.wisc.edu/ ece533/images/. 